\title{
The nonlocal Cahn-Hilliard-Hele-Shaw system with logarithmic potential
}

\author{
Francesco Della Porta ${ }^{1}$, Andrea Giorgini ${ }^{2}$ and Maurizio Grasselli ${ }^{3}$ \\ 1 Mathematical Institute, University of Oxford, Oxford OX2 6GG, United Kingdom \\ 2 Dipartimento di Matematica, Università degli Studi di Pavia, Pavia 27100, Italy \\ 3 Dipartimento di Matematica, Politecnico di Milano, Milano 20133, Italy \\ E-mail: dellaporta@maths.ox.ac.uk, andrea.giorgini@unipv.it \\ and maurizio.grasselli@polimi.it
}

Received 24 October 2017, revised 23 June 2018

Accepted for publication 23 July 2018

Published 12 September 2018

Recommended by Professor Bruno Eckhardt

\begin{abstract}
We study a diffuse interface model of the two-component Hele-Shaw flow. This is an advective Cahn-Hilliard equation for the relative concentration $\varphi$, where the incompressible velocity field $\boldsymbol{u}$ is determined by the Darcy's law depending on the Korteweg force $\mu \nabla \varphi$. Here $\mu$ is the derivative of a nonlocal non-convex free energy characterized by a logarithmic potential. The system is subject to no-flux boundary conditions for $\boldsymbol{u}$ and $\mu$ along with an initial condition for $\varphi$. First of all, we prove that the corresponding problem is globally well posed with respect to a natural notion of weak solution. Also, we establish the existence of global strong solutions. In dimension two, we show the validity of the so-called instantaneous separation property. This means that any solution, which is not a pure phase initially, stays away from the pure phases, uniformly with respect to the initial energy and total mass. Finally we prove the existence of the global attractor for the corresponding dynamical system as well as the convergence to a single equilibrium of any weak solution in the two-dimensional case.
\end{abstract}

Keywords: Cahn-Hilliard equation, Darcy's law, logarithmic potential, uniqueness, strong solutions and regularity, longtime behavior Mathematics Subject Classification numbers: 35B40, 35B65, 35D35, 35Q35

This is the Accepted Manuscript version of an article accepted for publication in Nonlinearity, 2018, 31:10, 4851-4881. IOP Publishing Ltd is not responsible for any errors or omissions in this version of the manuscript or any version derived from it. The version of record is available online at: https://doi.org/10.1088/1361-6544/aad52a 


\section{Introduction}

The motion of a binary fluid mixture confined in a cell between two parallel plates, separated by a narrow gap, is commonly called Hele-Shaw flow. When temperature gradients or shear stresses act along the interface between different components, driving respectively a thermocapillary and a mixing flow, the phase interfaces merge and reconnect in a transition towards an equilibrium configuration. The mathematical description of these evolutionary phenomena is a fundamental and challenging problem in the branch of hydrodynamics that studies topological changes observed at the interfaces between immiscible fluid mixtures. Within the diffuse interface approach, the above-mentioned processes are described by the Cahn-Hilliard-Hele-Shaw system proposed in [41] (see also [16, 38, 45]). This model has been deduced from the classical Cahn-Hilliard-Navier-Stokes system (also known as 'model $\mathrm{H}^{\prime}$ ) for the motion of an incompressible and immiscible fluid mixture by assuming that the viscous forces prevail over the inertial ones and the flow is of Poiseuille type. Denoting the (volume-averaged) velocity field by $\boldsymbol{u}$ and the difference of the fluid concentrations by $\varphi$, in the case of matched densities (neglecting also the gravity, see section 7), the system reads as follows

$$
\left\{\begin{array}{l}
\boldsymbol{u}=-\nabla P+\mu \nabla \varphi, \\
\operatorname{div} \boldsymbol{u}=0, \\
\varphi_{t}+\boldsymbol{u} \cdot \nabla \varphi-\Delta \mu=0, \\
\mu=-\Delta \varphi+\Psi^{\prime}(\varphi),
\end{array}\right.
$$

where $\Omega$ is a bounded domain in $\mathbb{R}^{d}, d=2,3$, with a sufficiently smooth boundary. Here all the physical constants have been set equal to one, $P$ stands for the pressure and $\Psi$ is a suitable double well potential. The physically most relevant choice of $\Psi$ is given by the logarithmic potential (see [8], see also $[9,37]$ and the references therein), namely,

$\Psi(s)=\frac{\theta}{2}[(1+s) \log (1+s)+(1-s) \log (1-s)]-\frac{\theta_{0}}{2} s^{2}, \quad \forall s \in(-1,1)$,

where $\theta>0$ and $\theta_{0}>0$ represent, respectively, the absolute and the critical temperature (both supposed to be constant). If $\theta_{0}>\theta$ then $\Psi$ is nonconvex with two symmetric wells. It is worth pointing out that the Cahn-Hilliard-Hele-Shaw system is originally a twodimensional model [16, 42]. However, the same system can be formally derived to describe porous media flows in three dimensions see, for instance, [33]. For this reason, the CahnHilliard-Hele-Shaw system is sometimes referred to as the Cahn-Hilliard-Darcy system in certain literature (see e.g. $[18,36]$ ).

System (1.1) with (1.2) and subject to no-flux boundary conditions has been analyzed in [27]. The existence of a global-in-time weak solution has been proven (see also [15] for a more general system). In dimension two, the uniqueness of weak solutions as well as the instantaneous propagation of regularity have been established. Then, the validity of the socalled instantaneous (also known as strict or uniform) separation property has been shown. This means that $\varphi$ stays instantaneously away from the pure states \pm 1 (uniformly with respect to the initial energy and the total mass), provided that the initial condition $\varphi_{0}$ is not a pure state. In dimension three, the existence of a (unique) global-in-time strong solution close to a given local minimizers of the free energy has been demonstrated. In addition, the convergence to a single equilibrium has been proven though, in dimension three, the result holds provided that the initial datum is close to a local minimizer of the free energy. Comparing this theoretical picture with the results obtained in the regular potential case (e.g. 
$\left.\Psi(s)=\frac{1}{4}\left(s^{2}-1\right)^{2}, s \in \mathbb{R}\right)$, we can say that the former is richer (see [6, 18, 36, 43, 46, 47], see also [16] for unmatched densities). Nonetheless, system (1.1) is difficult to handle, especially in dimension three. For instance, in the latter case, no uniqueness of weak solutions or general existence of global-in-time strong solutions are known. Roughly speaking, system (1.1) does not appear to be any simpler than the corresponding Cahn-Hilliard-NavierStokes system (see [1]).

The Cahn-Hilliard equation is well grounded from the physical viewpoint. More precisely, it can be viewed as a conserved gradient flow associated with the (non-convex) GinzburgLandau free energy

$$
\mathcal{U}(\varphi)=\int_{\Omega} \frac{1}{2}|\nabla \varphi(x)|^{2}+\Psi(\varphi(x)) \mathrm{d} x
$$

On the other hand, going back to the classical literature (see [3] and the references therein), the non-zero thickness in diffuse interface theories is not postulated as arbitrary, but rather connected with the finite range interaction between molecules. This aspect leads to nonlocal effects in the free energy (see [25]). However, these nonlocal interactions are generally described through a dependence on local concentration gradients in the energy (see (1.3)). Thus, in the free energy (1.3) the nonlocal effects are approximated. The same argument also goes for the Cahn-Hilliard-Hele-Shaw system, as recently illustrated in [48], where (1.1) has been employed to model solid tumor growth (see also [43] and the references therein). In particular, the starting adhesion energy has the form

$$
\mathcal{E}(\varphi)=\int_{\Omega} F(\varphi(x)) \mathrm{d} x-\frac{1}{2} \int_{\Omega} \int_{\Omega} J(x-y) \varphi(x) \varphi(y) \mathrm{d} x \mathrm{~d} y,
$$

where $J$ is a suitable interaction kernel and $F$ is related to the entropy of mixing, namely

$$
F(s)=\frac{\theta}{2}[(1+s) \log (1+s)+(1-s) \log (1-s)], \quad \forall s \in(-1,1) .
$$

This is exactly the free energy associated with the so-called nonlocal Cahn-Hilliard equation with logarithmic potential (see [24] and the references therein). We recall that this macroscopic energy can be derived from a microscopic model through a hydrodynamic limit (see [25]).

In this work, on account of the above considerations, we aim to study a variant of (1.1) by replacing the standard Cahn-Hilliard equation with its nonlocal version. More precisely, we consider the system

$$
\left\{\begin{array}{l}
\boldsymbol{u}=-\nabla P+\mu \nabla \varphi, \\
\operatorname{div} \boldsymbol{u}=0, \\
\varphi_{t}+\boldsymbol{u} \cdot \nabla \varphi-\Delta \mu=0, \\
\mu=F^{\prime}(\varphi)-J * \varphi
\end{array}\right.
$$

where $\Omega \subset \mathbb{R}^{d}$ with $d=2,3$ is bounded and smooth, $J: \mathbb{R}^{d} \rightarrow \mathbb{R}$ is such that $J(x)=J(-x)$, the convolution product stands for

$$
(J * \varphi)(x)=\int_{\Omega} J(x-y) \varphi(y) \mathrm{d} y,
$$

and $F$ is the convex logarithmic potential (1.5) (or a more general potential satisfying assump-tions (H.2) below). System (1.6) is subject to the following natural boundary and initial conditions 


$$
\begin{cases}\boldsymbol{u} \cdot \boldsymbol{n}=\frac{\partial \mu}{\partial \boldsymbol{n}}=0, & \text { on } \partial \Omega \times(0, T), \\ \varphi(\cdot, 0)=\varphi_{0}, & \text { in } \Omega,\end{cases}
$$

where $\boldsymbol{n}$ is the unit outward normal vector to the boundary $\partial \Omega$. We observe, in particular, that this system is the cornerstone of the model discussed and then approximated in [48].

From the mathematical viewpoint, passing from a fourth order equation to a second order equation entails some advantages, provided one knows how to handle the lack of regularity (see [17] for the regular potential case, see also [19-22, 24] for the Cahn-Hilliard-NavierStokes system). The goal of this paper is to show that we can prove uniqueness and regularity results in dimension three for system (1.6) and (1.7), which seem out of reach for system (1.1) with a singular potential $\Psi$ like (1.2). We first prove the existence of global-intime weak solution via the energy method. This is mainly based on the dissipative nature of the system given by the (formal) energy identity

$$
\mathcal{E}(\varphi(t))+\int_{0}^{t}\|\nabla \mu(\tau)\|^{2}+\|\boldsymbol{u}(\tau)\|^{2} \mathrm{~d} \tau=\mathcal{E}\left(\varphi_{0}\right), \quad \forall t \geqslant 0 .
$$

Thanks to an extra regularity for $\boldsymbol{u}$, obtained by carefully rewriting the Korteweg force, we establish that the global-in-time weak solution depends on the initial datum continuously, and so it is unique. Then, we establish the existence of strong solutions under a further natural assumption on the initial datum. By virtue of the dissipativity, this entails that any weak solution becomes instantaneously a strong one. Further regularity properties are also achieved in two space dimensions on account of the validity of the instantaneous separation property. In the final part, we discuss the longtime behavior. More precisely, the smoothing effect of any solution implies the existence of the global attractor as well as the convergence (in dimension two) of any weak solution to a unique equilibrium. We conclude by observing that, comparing the results obtained so far for various kinds of Cahn-Hilliard-Hele-Shaw models, the nonlocal case with logarithmic potential is the one with the richest picture in terms of wellposedness and regularity in dimension three. Therefore, at least in this case, avoiding approximations seems to be a good choice from the theoretical viewpoint.

\subsection{Plan of the paper}

In section 2 we recall the mathematical framework we will need throughout our analysis. Section 3 is devoted to existence and uniqueness of weak solution. The existence of strong solutions is discussed in section 4. Further regularity properties in two dimensions are provided in section 5. Finally, the content of section 6 is concerned with the asymptotic behavior.

\section{Notation and functional spaces}

We indicate by $W^{k, p}(\Omega), k \in \mathbb{N}$, the Sobolev space of real valued $L^{p}(\Omega)$-functions whose distributional derivatives of order less or equal to $k$ belong to $L^{p}(\Omega)$. Its norm is denoted by

$\|\cdot\|_{W^{k, p}(\Omega)}$. For an arbitrary $k \in \mathbb{N}, H^{k}(\Omega)=W^{k, 2}(\Omega)$ is a Hilbert space. The vector spaces $\left[W^{k, p}(\Omega)\right]^{d}$ endowed with the product structure ( $d$ is the spatial dimension) is denoted by $\mathbf{W}^{k, p}(\Omega)$. As customary we set $H=L^{2}(\Omega)$ and the inner product as well as the norm in $H$ are denoted by $(\cdot, \cdot)$ and $\|\cdot\|$, respectively. We also set $V=H^{1}(\Omega)$. We recall the following wellknown interpolation inequalities

$$
\|v\|_{L^{4}(\Omega)} \leqslant C\|v\|^{1-\frac{d}{4}}\|v\|_{V}^{\frac{d}{4}}, \quad \forall v \in V, \text { if } d=2,3,
$$




$$
\|v\|_{L^{3}(\Omega)} \leqslant C\|v\|^{\frac{1}{2}}\|v\|_{V}^{\frac{1}{2}}, \quad \forall v \in V, \text { if } d=3 .
$$

We indicate by $V^{\prime}$ the dual space of $V$. It is endowed with the standard dual norm $\|\cdot\|_{V^{\prime}}$ and $\langle\cdot, \cdot\rangle$ denotes the corresponding duality product. For every $f \in V^{\prime}$, we denote the total mass of $f$ over $\Omega$ by $\bar{f}=\frac{1}{|\Omega|}\langle f, 1\rangle$. We introduce the spaces of zero-mass functions

$$
V_{0}=\{v \in V: \bar{v}=0\}, \quad L_{0}^{2}=\{v \in H: \bar{v}=0\}, \quad V_{0}^{\prime}=\left\{f \in V^{\prime}: \bar{f}=0\right\}
$$

and the weak Neumann operator $A \in \mathcal{L}\left(V, V^{\prime}\right)$ defined by

$$
\langle A u, v\rangle=\int_{\Omega} \nabla u \cdot \nabla v \mathrm{~d} x, \quad \forall u, v \in V .
$$

The restriction of $A$ in $V_{0}$ is an isomorphism from $V_{0}$ onto $V_{0}^{\prime}$. We define the inverse map $\mathcal{N}: V_{0}^{\prime} \rightarrow V_{0}$ such that $A \mathcal{N} f=f$, for all $f \in V_{0}^{\prime}$, and $\mathcal{N} A v=v$, for all $v \in V_{0}$. In accordance with these definitions, we equip $V_{0}^{\prime}$ with the norm $\|f\|_{V_{0}^{\prime}}=\|\nabla \mathcal{N} f\|$ and we recall the chain rule

$$
\left\langle f_{t}, \mathcal{N} f\right\rangle=\frac{1}{2} \frac{\mathrm{d}}{\mathrm{d} t}\|f\|_{V_{0}^{\prime}}^{2} \quad \text { a.e. } t \in(0, T), \forall f \in H^{1}\left(0, T ; V_{0}^{\prime}\right)
$$

For every $f \in V^{\prime}$, we set $\|f\|_{*}=\left(\|f-\bar{f}\|_{V_{0}^{\prime}}^{2}+|\bar{f}|^{2}\right)^{\frac{1}{2}}$. It is well known that $\|\cdot\|_{*}$ is a norm on $V^{\prime}$ which is equivalent to the usual norm. We report the following elliptic estimates for the Neumann problem

$$
\|\nabla \mathcal{N} f\|_{\mathbf{H}^{k}(\Omega)} \leqslant C\|f\|_{H^{k-1}(\Omega)}, \quad \forall f \in H^{k-1}(\Omega) \cap L_{0}^{2}(\Omega), k=1,2 .
$$

We introduce the Hilbert space of solenoidal vector fields

$$
\mathbf{H}_{\sigma}=\left\{\mathbf{v} \in \mathbf{H}: \operatorname{div} \mathbf{v}=0,\left.\mathbf{v} \cdot \boldsymbol{n}\right|_{\partial \Omega}=0\right\} .
$$

In the sequel $(\cdot, \cdot)$ and $\|\cdot\|$ will also indicate the norm and the inner product in $\mathbf{H}_{\sigma}$, respectively. We denote by $\Pi$ the Helmholtz-Leray orthogonal projection from $\mathbf{H}$ onto $\mathbf{H}_{\sigma}$. We recall that every vector field $\boldsymbol{u} \in \mathbf{H}$ can be uniquely represented as

$$
\boldsymbol{u}=\mathbf{v}+\nabla P,
$$

where $\mathbf{v}=\Pi \boldsymbol{u} \in \mathbf{H}_{\sigma}$ and $P \in V_{0}$. Moreover, $\Pi$ is a bounded operator from $\mathbf{W}^{k, p}(\Omega)(k \in \mathbb{N}, 1$ $<p<\infty$ ) into itself (see [26, lemma 3.3]), namely

$$
\|\Pi \boldsymbol{u}\|_{\mathbf{W}^{k, p}(\Omega)} \leqslant C\|\boldsymbol{u}\|_{\mathbf{W}^{k, p}(\Omega)}, \quad \forall \boldsymbol{u} \in \mathbf{W}^{k, p}(\Omega),
$$

where the constant $C$ depends on $p$. On the other hand, we infer from $\nabla P=\boldsymbol{u}-\Pi \boldsymbol{u}$ that $P$ is the unique solution of the Neumann problem

$$
\begin{cases}-\Delta P=\operatorname{div} \boldsymbol{u}, & \text { in } \Omega, \\ \frac{\partial P}{\partial \boldsymbol{n}}=\boldsymbol{u} \cdot \boldsymbol{n}, & \text { on } \partial \Omega,\end{cases}
$$

such that $\bar{P}=0$. By the classical elliptic regularity for the Neumann problem, given $\boldsymbol{u} \in \mathbf{W}^{k, p}(\Omega)$, it follows that $P \in W^{k+1, p}(\Omega)$. In addition, solenoidal vector fields $\operatorname{in} \mathbf{V} \cap \mathbf{H}_{\sigma}$ fulfill the following inequality (see [28, theorem 3.8])

$$
\|\mathbf{v}\|_{\mathbf{v}} \leqslant C(\|\nabla \times \mathbf{v}\|+\|\mathbf{v}\|), \quad \forall \mathbf{v} \in \mathbf{V} \cap \mathbf{H}_{\sigma} .
$$


We shall also make use of the higher-order solenoidal Hilbert space

$$
\mathbf{V}_{\sigma}=\left\{\mathbf{v} \in \mathbf{V}: \operatorname{div} \mathbf{v}=0,\left.\mathbf{v}\right|_{\partial \Omega}=0\right\}
$$

equipped with inner product $\langle\boldsymbol{u}, \mathbf{v}\rangle_{\mathbf{v}_{\sigma}}=(\nabla \boldsymbol{u}, \nabla \mathbf{v})$ and norm $\|\boldsymbol{u}\|_{\mathbf{v}_{\sigma}}=\|\nabla \boldsymbol{u}\|$.

Throughout the paper, if not specified otherwise, we indicate by $C$ generic positive constants depending only on structural quantities. The constants $C$ may vary from line to line increasing their value. Any further dependence will be explicitly pointed out if necessary.

\section{Well-posedness}

In this section we prove that problem (1.6) and (1.7) is well posed with respect to the notion of weak solutions in $\Omega \subset \mathbb{R}^{3}$. The given functions $J$ and $F$ are supposed to fulfill the main assumptions:

(H.1) $\quad J \in W^{1,1}\left(\mathbb{R}^{d}\right)$ with $J(x)=J(-x)$;

(H.2) $\quad F \in \mathcal{C}([-1,1]) \cap \mathcal{C}^{2}(-1,1)$ such that

$$
\lim _{s \rightarrow-1} F^{\prime}(s)=-\infty, \quad \lim _{s \rightarrow 1} F^{\prime}(s)=+\infty \quad \text { and } \quad F^{\prime \prime}(s) \geqslant \alpha>0 .
$$

Without loss of generality, we require that $F(s)=+\infty$, for all $s \in[-1,1]^{c}$, and $F(0)=F^{\prime}(0)=0$.

Remark 3.1. Condition (H.2) is satisfied and motivated by the logarithmic potential given by (1.5).

By weak solution we mean the following

Definition 3.1. Let $\varphi_{0}: \Omega \rightarrow \mathbb{R}$ be a measurable function with $F\left(\varphi_{0}\right) \in L^{1}(\Omega),\left|\bar{\varphi}_{0}\right|<1$ and $T>0$ be given. A triple $(\boldsymbol{u}, P, \varphi)$ is a weak solution to problem (1.6) and (1.7) on $[0, T]$ corresponding to $\varphi_{0}$ if

$$
\begin{aligned}
& \boldsymbol{u} \in L^{2}\left(0, T ; \mathbf{H}_{\sigma}\right), \quad P \in L^{1}\left(0, T ; W^{1, \frac{3}{2}}(\Omega)\right), \\
& \varphi \in L^{\infty}(0, T ; H) \cap L^{2}(0, T ; V) \cap H^{1}\left(0, T ; V^{\prime}\right), \\
& \varphi \in L^{\infty}(\Omega \times(0, T)) \text { with }|\varphi(x, t)|<1 \text { a.e. }(x, t) \in \Omega \times(0, T), \\
& F(\varphi) \in L^{\infty}\left(0, T ; L^{1}(\Omega)\right), \quad F^{\prime}(\varphi) \in L^{2}(0, T ; V), \\
& \mu \in L^{2}(0, T ; V),
\end{aligned}
$$

such that

$$
\left\langle\varphi_{t}, v\right\rangle-(\boldsymbol{u} \varphi, \nabla v)+(\nabla \mu, \nabla v)=0, \quad \forall v \in V, \text { a.e. in }(0, T)
$$

where

$$
\boldsymbol{u}=-\nabla P+\mu \nabla \varphi, \quad \mu=F^{\prime}(\varphi)-J * \varphi, \quad \text { a.e. in } \Omega \times(0, T)
$$

and satisfies $\varphi(0, \cdot)=\varphi_{0}$ a.e. in $\Omega$.

Remark 3.2. We deduce from the assumptions $F\left(\varphi_{0}\right) \in L^{1}(\Omega)$ and $\left|\bar{\varphi}_{0}\right|<1$ that the class of admissible initial conditions consist of $\varphi_{0} \in L^{\infty}(\Omega)$ such that $\left|\varphi_{0}(x)\right| \leqslant 1$, for almost every $x \in \Omega$. However, they cannot be pure phases, namely $\varphi_{0} \not \equiv \pm 1$, due to the restriction on the total mass. In addition, concerning the initial condition, note that $\varphi \in \mathcal{C}([0, T], H)$. Also, any 
weak solution satisfies the mass conservation property, namely

$$
\bar{\varphi}(t)=\bar{\varphi}_{0}, \quad \forall t \geqslant 0 .
$$

Remark 3.3. On account of the regularity satisfied by $\varphi$, any weak solution fulfills the identity

$$
\mu \nabla \varphi=\nabla(F(\varphi)-(J * \varphi) \varphi)+(\nabla J * \varphi) \varphi
$$

almost everywhere in $\Omega \times(0, T)$. Thus, the Darcy's law is equivalent to

$$
\boldsymbol{u}=-\nabla P^{*}+(\nabla J * \varphi) \varphi, \quad P^{*}=P-F(\varphi)+(J * \varphi) \varphi, \quad \text { a.e. in } \Omega \times(0, T),
$$

and its weak formulation becomes

$$
\int_{\Omega} \boldsymbol{u} \cdot \boldsymbol{v} \mathrm{d} x=\int_{\Omega}(\nabla J * \varphi) \varphi \cdot \boldsymbol{v} \mathrm{d} x, \quad \forall \boldsymbol{v} \in \mathbf{H}_{\sigma}, \text { a.e. in }(0, T) .
$$

In particular, the modified pressure $P^{*} \in L^{2}\left(0, T ; V_{0}\right)$. However, since the product $\mu \nabla \varphi$ is bounded in $L^{1}\left(0, T ; \mathbf{L}^{\frac{3}{2}}(\Omega)\right)$, the original pressure $P$ belongs to $L^{1}\left(0, T ; W^{1, \frac{3}{2}}(\Omega)\right)$.

Our first result regarding the existence of a weak solution is given by

Theorem 3.1. Let $\varphi_{0}$ be a measurable function with $F\left(\varphi_{0}\right) \in L^{1}(\Omega)$ and $\left|\bar{\varphi}_{0}\right|<1$. Then, there exists at least a weak solution $(\boldsymbol{u}, P, \varphi)$ to problem (1.6) and (1.7) satisfying

$$
\sup _{t \geqslant 0}\|\varphi(t)\|_{L^{\infty}(\Omega)} \leqslant 1 .
$$

In addition, assuming that $\left|\bar{\varphi}_{0}\right| \leqslant m$ for some $m \in[0,1)$, there exists $C=C(m)>0$, independent of the initial datum, such that

$$
\|\boldsymbol{u}\|_{L^{2}\left(t, t+1 ; \mathbf{H}_{\sigma}\right)}+\|\mu\|_{L^{2}(t, t+1 ; V)}+\|\varphi\|_{L^{2}(t, t+1 ; V)} \leqslant C, \quad \forall t \geqslant 0 .
$$

A solution in the sense of definition 3.1 will be constructed through several approximating problems. We introduce an artificial viscosity $-\varepsilon \Delta \boldsymbol{u}$, with $\varepsilon>0$, in the Darcy's law and we replace the singular potential with a family of regular ones $F_{\lambda}$, depending on the positive parameter $\lambda$, which converges in a suitable way to $F$ and satisfy some uniform properties with respect to $\lambda$. In this framework, the existence of a pair $\left(\boldsymbol{u}^{\varepsilon, \lambda}, \varphi^{\varepsilon, \lambda}\right)$ is carried out via the standard Galerkin scheme (the pressure will be recovered at the end of this argument). Then we aim to derive some uniform estimates with respect to the approximation parameters $\lambda$ and $\varepsilon$. After that, it will be convenient to pass to the limit as $\lambda$ which goes to 0 , with $\varepsilon>0$ fixed. In such a way, the velocity field is bounded in $L^{2}\left(0, T ; \mathbf{V}_{\sigma}\right)$ at this stage. This facilitates the goal of finding an estimate for $\varphi_{t}^{\varepsilon, \lambda}$. Next, taking advantage of the uniform bound in $L^{\infty}(\Omega \times(0, T))$ of $\varphi^{\varepsilon}$, we recover the limit system via compactness letting go to 0 . We refer the reader to $[17,20,24]$ and the references therein for some arguments used in the sequel.

Proof. The proof will be divided into five steps.

\subsection{A two levels approximation problem}

For any given $\varepsilon>0$ and $\lambda>0$, we consider the Cahn-Hilliard-Brinkman (CHB) system (see [17], c.f. also [6,14] and the references therein) 


$$
\left\{\begin{array}{l}
-\varepsilon \Delta \boldsymbol{u}+\boldsymbol{u}=-\nabla P+\mu \nabla \varphi, \\
\operatorname{div} \boldsymbol{u}=0, \\
\varphi_{t}+\boldsymbol{u} \cdot \nabla \varphi-\Delta \mu=0, \\
\mu=F_{\lambda}^{\prime}(\varphi)-J * \varphi,
\end{array} \quad \text { in } \Omega \times(0, T),\right.
$$

subject to the following boundary and initial conditions

$$
\begin{cases}\boldsymbol{u}=\mathbf{0}, \quad \frac{\partial \mu}{\partial \boldsymbol{n}}=0, & \text { on } \partial \Omega \times(0, T), \\ \varphi(\cdot, 0)=\varphi_{0}, & \text { in } \Omega .\end{cases}
$$

The family olf regular functions $F_{\lambda}: \mathbb{R} \rightarrow \mathbb{R}$ can be constructed in such a way that

(i) for any $0<\bar{\lambda} \leqslant 1$, there exists $C>0$ such that

$$
F_{\lambda}(s) \geqslant \frac{1}{4 \bar{\lambda}} s^{2}-C, \quad \forall s \in \mathbb{R}, \forall \lambda \in(0, \bar{\lambda}] ;
$$

(ii) $F_{\lambda}$ is convex with

$$
F_{\lambda}^{\prime \prime}(s) \geqslant \frac{\alpha}{1+\alpha}, \quad \forall s \in \mathbb{R}
$$

(iii) $F_{\lambda}^{\prime}$ is Lipschitz on $\mathbb{R}$ with constant $\frac{1}{\lambda}$;

(iv) $F_{\lambda}(s) \nearrow F(s)$ and $\left|F_{\lambda}^{\prime}(s)\right| \nearrow\left|F^{\prime}(s)\right|$ for every $s \in \mathbb{R}$ as $\lambda \rightarrow 0$ and, in addition, $F_{\lambda}^{\prime}$ converges uniformly to $F^{\prime}$ on any interval $[a, b] \subset(-1,1)$;

(v) $F_{\lambda}(0)=F_{\lambda}^{\prime}(0)=0$, for all $\lambda>0$.

The existence of such a family of regular potentials has been shown in [24] appealing to the theory of maximal monotone operators and according to the assumptions on the singular potential (H.2).

As previously anticipated, arguing as in [17] and [24], one can prove the exist-ence of a weak solution to problem (3.6) and (3.7) via a Galerkin scheme, by exploit-ing standard energy estimates and then passing to the limit in the usual way. More precisely, given an initial datum $\varphi_{0} \in H$, there exists a pair $(\boldsymbol{u}, \varphi)$ such that $\boldsymbol{u} \in L^{2}\left(0, T ; \mathbf{V}_{\sigma}\right)$, $\varphi \quad \in L^{\infty}(0, T ; H) \cap L^{2}(0, T ; V)$ with time derivative $e_{t} \in L^{\frac{4}{3}}\left(0, T ; V^{\prime}\right)$, which satisfies (see remark 3.3)

$$
\begin{aligned}
& \varepsilon(\nabla \boldsymbol{u}, \nabla \boldsymbol{v})+(\boldsymbol{u}, \boldsymbol{v})=((\nabla J * \varphi) \varphi, \boldsymbol{v}), \forall \boldsymbol{v} \in \mathbf{V}_{\sigma}, \text { a.e. } t \in(0, T), \\
& \left\langle\varphi_{t}, v\right\rangle-(\boldsymbol{u} \varphi, \nabla v)+(\nabla \mu, \nabla v)=0, \quad \forall v \in V, \text { a.e. } t \in(0, T),
\end{aligned}
$$

where $\mu=F_{\lambda}^{\prime}(\varphi)-J * \varphi \in L^{2}(0, T ; V)$. Furthermore, introducing the regularized free energy

$$
\mathcal{E}_{\lambda}(\varphi)=\int_{\Omega} F_{\lambda}(\varphi(x)) \mathrm{d} x-\frac{1}{2} \int_{\Omega} \int_{\Omega} J(x-y) \varphi(x) \varphi(y) \mathrm{d} x \mathrm{~d} y, \quad \forall \varphi \in H,
$$

the energy inequality holds

$$
\mathcal{E}_{\lambda}(\varphi(t))+\int_{0}^{t} \varepsilon\|\nabla \boldsymbol{u}(\tau)\|^{2}+\|\boldsymbol{u}(\tau)\|^{2}+\|\nabla \mu(\tau)\|^{2} \mathrm{~d} \tau \leqslant \mathcal{E}_{\lambda}\left(\varphi_{0}\right),
$$

for almost every $t \geqslant 0$. 


\subsection{A priori bounds based on the energy estimate}

We consider an admissible initial datum such that $\varphi_{0}$ is measurable with $F\left(\varphi_{0}\right) \in L^{1}(\Omega)$ and $\left|\bar{\varphi}_{0}\right|<1$. According to the previous stage, for any $\varepsilon>0$ and $\lambda>0$, there exists a pair $\left(\boldsymbol{u}^{\varepsilon, \lambda}, \varphi^{\varepsilon, \lambda}\right)$ satisfying the weak formulation (3.10) and (3.11) and the energy inequality (3.12). Our next goal is to derive some a priori uniform bounds with respect to $\varepsilon$ and $\lambda$.

By virtue of the properties of $F_{\lambda}$, we deduce that $\mathcal{E}_{\lambda}\left(\varphi_{0}\right) \leqslant \mathcal{E}\left(\varphi_{0}\right)$. In light of (3.12), we find

$\mathcal{E}_{\lambda}\left(\varphi^{\varepsilon, \lambda}(t)\right)+\int_{0}^{t} \varepsilon\left\|\boldsymbol{u}^{\varepsilon, \lambda}(\tau)\right\|_{\mathbf{V}_{\sigma}}^{2}+\left\|\boldsymbol{u}^{\varepsilon, \lambda}(\tau)\right\|^{2}+\left\|\nabla \mu^{\varepsilon, \lambda}(\tau)\right\|^{2} \mathrm{~d} \tau \leqslant \mathcal{E}\left(\varphi_{0}\right)$

for almost every $t \in[0, T]$. We notice that, as a consequence of property (i), we have in particular

$\left\|\varphi^{\varepsilon, \lambda}(t)\right\|^{2}+\int_{0}^{t} \varepsilon\left\|\boldsymbol{u}^{\varepsilon, \lambda}(\tau)\right\|_{\mathbf{V}_{\sigma}}^{2}+\left\|\boldsymbol{u}^{\varepsilon, \lambda}(\tau)\right\|^{2}+\left\|\nabla \mu^{\varepsilon, \lambda}(\tau)\right\|^{2} \mathrm{~d} \tau \leqslant \mathcal{E}\left(\varphi_{0}\right)+C$,

for almost every $0 \leqslant t \leqslant T$, with $C$ independent of $\varepsilon$ and $\lambda$. Taking the gradient of $\mu^{\varepsilon, \lambda}$ and testing by $\nabla \varphi^{\varepsilon, \lambda}$, we also obtain

$$
\left(F_{\lambda}^{\prime \prime}\left(\varphi^{\varepsilon, \lambda}\right) \nabla \varphi^{\varepsilon, \lambda}, \nabla \varphi^{\varepsilon, \lambda}\right)=\left(\nabla \mu^{\varepsilon, \lambda}, \nabla \varphi^{\varepsilon, \lambda}\right)+\left(\nabla J * \varphi^{\varepsilon, \lambda}, \nabla \varphi^{\varepsilon, \lambda}\right) .
$$

By property (ii) and the Young inequality for convolution product, we reach

$$
\left\|\nabla \varphi^{\varepsilon, \lambda}\right\|^{2} \leqslant C\left\|\nabla \mu^{\varepsilon, \lambda}\right\|^{2}+C\left\|\varphi^{\varepsilon, \lambda}\right\|^{2} .
$$

Thus, integrating in time and using (3.13) and (3.14), we arrive at

$$
\int_{0}^{T}\left\|\nabla \varphi^{\varepsilon, \lambda}(\tau)\right\|^{2} \mathrm{~d} \tau \leqslant C(1+T)\left(1+\mathcal{E}\left(\varphi_{0}\right)\right)
$$

We now prove a uniform estimate of $\mu^{\varepsilon, \lambda}$ in $V$. It is sufficient to find a control of the total mass $\bar{\mu}^{\varepsilon, \lambda}$, that is

$$
\bar{\mu}^{\varepsilon, \lambda}=\frac{1}{|\Omega|} \int_{\Omega} F_{\lambda}^{\prime}\left(\varphi^{\varepsilon, \lambda}\right) \mathrm{d} x-\frac{1}{|\Omega|} \int_{\Omega} J * \varphi^{\varepsilon, \lambda} \mathrm{d} x .
$$

Thanks to the monotonicity of $F_{\lambda}^{\prime}$, it is possible to show (see [20, equations (3.35)-3.37] or [24, proof of theorem 3.15]) that

$$
\left\|F_{\lambda}^{\prime}\left(\varphi^{\varepsilon, \lambda}\right)\right\|_{L^{1}(\Omega)} \leqslant C \int_{\Omega}\left(\varphi^{\varepsilon, \lambda}-\bar{\varphi}_{0}\right) F_{\lambda}^{\prime}\left(\varphi^{\varepsilon, \lambda}\right) \mathrm{d} x+C,
$$

where $C$ depends on $\bar{\varphi}_{0}$, but is independent of $\varepsilon$ and $\lambda$. Then, testing $\mu^{\varepsilon, \lambda}$ by $\varphi^{\varepsilon, \lambda}-\bar{\varphi}_{0}$ yields

$$
\int_{\Omega}\left(\varphi^{\varepsilon, \lambda}-\bar{\varphi}_{0}\right) F_{\lambda}^{\prime}\left(\varphi^{\varepsilon, \lambda}\right) \mathrm{d} x \leqslant C\left\|\nabla \mu^{\varepsilon, \lambda}\right\|\left\|\varphi^{\varepsilon, \lambda}-\bar{\varphi}_{0}\right\|+C\left\|\varphi^{\varepsilon, \lambda}\right\|^{2}+C .
$$

Collecting the above estimates and using (3.14), we find

$$
\int_{0}^{T}\left\|F_{\lambda}^{\prime}\left(\varphi^{\varepsilon, \lambda}\right)(\tau)\right\|_{L^{1}(\Omega)}^{2} \mathrm{~d} \tau \leqslant C(1+T)\left(1+\mathcal{E}\left(\varphi_{0}\right)\right)^{2},
$$

which, in turn, implies

$$
\int_{0}^{T}\left\|\mu^{\varepsilon, \lambda}(\tau)\right\|_{V}^{2} \mathrm{~d} \tau \leqslant C(1+T)\left(1+\mathcal{E}\left(\varphi_{0}\right)\right)^{2} .
$$

Observe that all the above estimates are independent of $\varepsilon$ and $\lambda$. 


\subsection{The $\mathrm{CHB}$ system with singular potential: the limit $\lambda \rightarrow 0^{+}$}

Our goal is to perform the limit $\lambda \rightarrow 0^{+}$. To this end, we need to derive a uniform control of $\varphi_{t}^{\varepsilon, \lambda}$. By comparison, using standard Sobolev embedding and (2.2), we have

$$
\begin{aligned}
\left\|\varphi_{t}^{\varepsilon, \lambda}\right\|_{V^{\prime}} & \leqslant\left\|\nabla \mu^{\varepsilon, \lambda}\right\|+\left\|\boldsymbol{u}^{\varepsilon, \lambda}\right\|_{\mathbf{L}^{6}(\Omega)}\left\|\varphi^{\varepsilon, \lambda}\right\|_{L^{3}(\Omega)} \\
& \leqslant\left\|\nabla \mu^{\varepsilon, \lambda}\right\|+C\left\|\boldsymbol{u}^{\varepsilon, \lambda}\right\|_{\mathbf{V}_{\sigma}}\left\|\varphi^{\varepsilon, \lambda}\right\|_{H}^{\frac{1}{2}}\left\|\varphi^{\varepsilon, \lambda}\right\|_{V^{\frac{1}{2}}}
\end{aligned}
$$

Hence, we get

$$
\begin{aligned}
\int_{0}^{T}\left\|\varphi_{t}^{\varepsilon, \lambda}(\tau)\right\|_{V^{\prime}}^{\frac{4}{3}} \mathrm{~d} \tau \leqslant & C \int_{0}^{T}\left(1+\left\|\nabla \mu^{\varepsilon, \lambda}\right\|^{2}\right) \mathrm{d} \tau+C\left\|\varphi^{\varepsilon, \lambda}\right\|_{L^{\infty}(0, T ; H)}^{\frac{2}{3}}\left(\int_{0}^{T}\left\|\boldsymbol{u}^{\varepsilon, \lambda}(\tau)\right\|_{\mathbf{V}_{\sigma}}^{2} \mathrm{~d} \tau\right)^{\frac{2}{3}} \\
& \left(\int_{0}^{T}\left\|\varphi^{\varepsilon, \lambda}(\tau)\right\|_{V}^{2} \mathrm{~d} \tau\right)^{\frac{1}{3}} \leqslant C(1+T)\left(1+\mathcal{E}\left(\varphi_{0}\right)\right)^{\frac{4}{3}}
\end{aligned}
$$

Here $C$ is independent of $\lambda$ but depends on $\varepsilon$. Collecting the above estimates, we deduce that

$$
\begin{gathered}
\left\|\varphi^{\varepsilon, \lambda}\right\|_{L^{\infty}(0, T ; H)} \leqslant C, \\
\left\|\varphi^{\varepsilon, \lambda}\right\|_{L^{2}(0, T ; V)} \leqslant C, \\
\left\|\varphi_{t}^{\varepsilon, \lambda}\right\|_{L^{\frac{4}{3}}\left(0, T ; V^{\prime}\right)} \leqslant C, \\
\left\|\boldsymbol{u}^{\varepsilon, \lambda}\right\|_{L^{2}\left(0, T ; \mathbf{V}_{\sigma}\right)} \leqslant C, \\
\left\|\mu^{\varepsilon, \lambda}\right\|_{L^{2}(0, T ; V)} \leqslant C,
\end{gathered}
$$

where the constant $C$ depends on the initial energy $\mathcal{E}\left(\varphi_{0}\right), \bar{\varphi}_{0}$, the form of $F$ and $\varepsilon$, but is independent of $\lambda$. Thanks to the uniform controls (3.19)-(3.23), letting $\lambda \rightarrow 0$, we deduce the following weak convergence results (up to subsequences)

$$
\begin{aligned}
& \varphi^{\varepsilon, \lambda} \rightarrow \varphi^{\varepsilon}, \quad \text { weakly star in } L^{\infty}(0, T ; H), \\
& \varphi^{\varepsilon, \lambda} \rightarrow \varphi^{\varepsilon}, \quad \text { weakly in } L^{2}(0, T ; V), \\
& \varphi_{t}^{\varepsilon, \lambda} \rightarrow \varphi_{t}^{\varepsilon}, \quad \text { weakly in } L^{\frac{4}{3}}\left(0, T ; V^{\prime}\right), \\
& \boldsymbol{u}^{\varepsilon, \lambda} \rightarrow \boldsymbol{u}^{\varepsilon}, \quad \text { weakly in } L^{2}\left(0, T ; \mathbf{V}_{\sigma}\right), \\
& \mu^{\varepsilon, \lambda} \rightarrow \mu^{\varepsilon}, \quad \text { weakly in } L^{2}(0, T ; V) .
\end{aligned}
$$

Besides, according to (3.20) and (3.21), an application of the Aubin-Lions compactness lemma entails

$$
\varphi^{\varepsilon, \lambda} \rightarrow \varphi^{\varepsilon}, \quad \text { strongly in } L^{2}\left(0, T ; L^{p}(\Omega)\right),
$$

for $p \in[2,6)$. In turn, this gives the pointwise convergence (up to subsequences)

$$
\varphi^{\varepsilon, \lambda} \rightarrow \varphi^{\varepsilon}, \quad \text { a.e. in } \Omega \times(0, T) .
$$

On account of the monotonicity of $F^{\prime}$ and the uniform bound (3.17), it is possible to show (see the argument in [20] and [24]) that the limit function $\varphi^{\varepsilon}$ fulfills 


$$
\varphi^{\varepsilon} \in L^{\infty}(\Omega \times(0, T)) \text { such that }\left|\varphi^{\varepsilon}(x, t)\right|<1 \text { a.e. in } \Omega \times(0, T) .
$$

As a consequence, we deduce from property (iv) and the pointwise convergence (3.30) that

$$
F_{\lambda}^{\prime}\left(\varphi^{\varepsilon, \lambda}\right) \rightarrow F^{\prime}\left(\varphi^{\varepsilon}\right), \quad \text { a.e. in } \Omega \times(0, T) .
$$

By comparison, we also get

$$
\left\|F_{\lambda}^{\prime}\left(\varphi^{\varepsilon, \lambda}\right)\right\|_{L^{2}(0, T ; V)} \leqslant C
$$

with $C$ independent of $\lambda$. Then, we infer that $F_{\lambda}^{\prime}\left(\varphi^{\varepsilon, \lambda}\right) \rightarrow \chi$ weakly in $L^{2}(0, T ; V)$. On the other hand, the pointwise convergence (3.32) and the uniform control of $F_{\lambda}^{\prime}\left(\varphi^{\varepsilon, \lambda}\right)$ in $L^{2}(0, T ; H)$ (with respect to $\lambda$ ) imply that $F_{\lambda}^{\prime}\left(\varphi^{\varepsilon, \lambda}\right) \rightarrow F^{\prime}\left(\varphi^{\varepsilon}\right)$ weakly in $L^{2}(0, T ; H)$. Hence, by the uniqueness of the weak limit

$$
F_{\lambda}^{\prime}\left(\varphi^{\varepsilon, \lambda}\right) \rightarrow F^{\prime}\left(\varphi^{\varepsilon}\right), \text { weakly in } L^{2}(0, T ; V) .
$$

Regarding the product terms, it follows from (H.1), (3.25) and (3.29) that

$$
\left(\nabla J * \varphi^{\varepsilon, \lambda}\right) \varphi^{\varepsilon, \lambda} \rightarrow\left(\nabla J * \varphi^{\varepsilon}\right) \varphi^{\varepsilon}, \quad \text { weakly in } L^{\frac{4}{3}}(0, T ; H)
$$

and

$$
\boldsymbol{u}^{\varepsilon, \lambda} \varphi^{\varepsilon, \lambda} \rightarrow \boldsymbol{u}^{\varepsilon} \varphi^{\varepsilon}, \quad \text { weakly in } L^{\frac{4}{3}}(0, T ; H) .
$$

Therefore, a passage to the limit in the weak formulation (3.34) and (3.35) yields

$\varepsilon\left(\nabla \boldsymbol{u}^{\varepsilon}, \nabla \boldsymbol{v}\right)+\left(\boldsymbol{u}^{\varepsilon}, \boldsymbol{v}\right)=\left(\left(\nabla J * \varphi^{\varepsilon}\right) \varphi^{\varepsilon}, \boldsymbol{v}\right), \quad \forall \boldsymbol{v} \in \mathbf{V}_{\sigma}$, a.e. $t \in(0, T)$,

$\left\langle\varphi_{t}^{\varepsilon}, v\right\rangle-\left(\boldsymbol{u}^{\varepsilon} \varphi^{\varepsilon}, \nabla v\right)+\left(\nabla \mu^{\varepsilon}, \nabla v\right)=0, \quad \forall v \in V$, a.e. $t \in(0, T)$,

where $\mu^{\varepsilon}=F^{\prime}\left(\varphi^{\varepsilon}\right)-J * \varphi^{\varepsilon}$ almost everywhere in $\Omega \times(0, T)$. Furthermore, owing to the above convergences, we can pass to the limit into the energy inequality (3.13) obtaining

$$
\mathcal{E}\left(\varphi^{\varepsilon}(t)\right)+\int_{0}^{t} \varepsilon\left\|\nabla \boldsymbol{u}^{\varepsilon}(\tau)\right\|^{2}+\left\|\boldsymbol{u}^{\varepsilon}(\tau)\right\|^{2}+\left\|\nabla \mu^{\varepsilon}(\tau)\right\|^{2} \mathrm{~d} \tau \leqslant \mathcal{E}\left(\varphi_{0}\right),
$$

for almost every $t \in[0, T]$, for any given $T>0$.

\subsection{The vanishing viscosity limit $\varepsilon \rightarrow 0^{+}$}

First, according to (3.31) and (3.36), it follows immediately that

$$
\begin{aligned}
& \left\|\varphi^{\varepsilon}\right\|_{L^{\infty}(\Omega \times(0, T))} \leqslant 1, \\
& \left\|\boldsymbol{u}^{\varepsilon}\right\|_{L^{2}\left(0, T ; \mathbf{H}_{\sigma}\right)} \leqslant C \\
& \left\|\nabla \mu^{\varepsilon}\right\|_{L^{2}(0, T ; H)} \leqslant C .
\end{aligned}
$$

Repeating line by line all the estimates performed in step 2, we arrive at

$$
\begin{aligned}
& \left\|\varphi^{\varepsilon}\right\|_{L^{2}(0, T ; V)} \leqslant C, \\
& \left\|\mu^{\varepsilon}\right\|_{L^{2}(0, T ; V)} \leqslant C, \\
& \left\|F^{\prime}\left(\varphi^{\varepsilon}\right)\right\|_{L^{2}(0, T ; V)} \leqslant C .
\end{aligned}
$$

We need to find a control for $\varphi_{t}$ that is independent of $\varepsilon$. Thanks to the uniform $L^{\infty}$-bound of $\varphi^{\varepsilon}$ (see also [24, remark 3.3]), we have by comparison 


$$
\left\|\varphi_{t}^{\varepsilon}\right\|_{V^{\prime}} \leqslant\left\|\nabla \mu^{\varepsilon}\right\|+\left\|\boldsymbol{u}^{\varepsilon}\right\|
$$

Hence, this leads to

$$
\left\|\varphi_{t}^{\varepsilon}\right\|_{L^{2}\left(0, T ; V^{\prime}\right)} \leqslant C .
$$

Being all the above bounds independent of $\lambda$, the following weak convergence results hold (up to subsequences)

$$
\begin{aligned}
& \varphi^{\varepsilon} \rightarrow \varphi, \quad \text { weakly star in } L^{\infty}(\Omega \times(0, T)), \\
& \varphi^{\varepsilon}-\varphi, \quad \text { weakly in } L^{2}(0, T ; V), \\
& \varphi_{t}^{\varepsilon}-\varphi_{t}, \quad \text { weakly in } L^{2}\left(0, T ; V^{\prime}\right), \\
& \boldsymbol{u}^{\varepsilon} \rightarrow \boldsymbol{u}, \quad \text { weakly in } L^{2}\left(0, T ; \mathbf{H}_{\sigma}\right), \\
& \mu^{\varepsilon} \rightarrow \mu, \quad \text { weakly in } L^{2}(0, T ; V) .
\end{aligned}
$$

We recall that, thanks to the Aubin-Lions lemma, we have that $\varphi^{\varepsilon} \rightarrow \varphi$ strongly in $L^{2}\left(0, T ; L^{p}(\Omega)\right)$ for any $p \in[2,6)$. Thanks to (3.37)-(3.41), and applying a similar argument to the one employed in step 3 , we are able to pass to the limit as $\varepsilon \rightarrow 0$ in the weak formulation (3.34) and (3.35) and the limit pair $(\boldsymbol{u}, \varphi)$ satisfies

$$
\begin{aligned}
& (\boldsymbol{u}, \boldsymbol{v})=((\nabla J * \varphi) \varphi, \boldsymbol{v}), \quad \forall v \in \mathbf{V}_{\sigma}, \text { a.e. in }(0, T), \\
& \left\langle\varphi_{t}, v\right\rangle-(\boldsymbol{u} \varphi, \nabla v)+(\nabla \mu, \nabla v)=0, \quad \forall v \in V, \text { a.e. in }(0, T),
\end{aligned}
$$

where $\mu=F^{\prime}(\varphi)-J * \varphi$. To conclude the proof, we need to comply with the weak formulation stated in definition 3.1 by recovering the pressure $P$. In this regard, by a density argument, we observe that $\boldsymbol{u}=\Pi((\nabla J * \varphi) \varphi)$. Thus, in accordance with (2.6), there exists $P^{*} \in L^{2}\left(0, T ; V_{0}\right)$ such that $\boldsymbol{u}=-\nabla P^{*}+(\nabla J * \varphi) \varphi$. Owing to remark 3.3, we conclude that $P=P^{*}+F(\varphi)-(J * \varphi) \varphi \in L^{1}\left(0, T ; W^{1, \frac{3}{2}}(\Omega)\right)$ and $\boldsymbol{u}=-\nabla P+\mu \nabla \varphi$. All identities here hold almost everywhere in $\Omega \times(0, T)$.

\subsection{Uniform dissipative estimates}

On account of the regularity (3.37)-(3.41), passing to the limit in (3.36) as $\varepsilon$ goes to 0 , we get

$$
\mathcal{E}(\varphi(t))+\int_{0}^{t}\|\boldsymbol{u}(\tau)\|^{2}+\|\nabla \mu(\tau)\|^{2} \mathrm{~d} \tau \leqslant \mathcal{E}\left(\varphi_{0}\right),
$$

for almost any $t \geqslant 0$. Since $\varphi \in \mathcal{C}([0, T], H)$, we deduce that $\int_{\Omega} F(\varphi(t)) \mathrm{d} x$ is bounded for any $t \geqslant 0$. In turn, it easily follows

$$
\sup _{t \geqslant 0}\|\varphi(t)\|_{L^{\infty}(\Omega)} \leqslant 1 .
$$

Moreover, due to the assumptions on the admissible initial datum, it is clear that $\mathcal{E}\left(\varphi_{0}\right) \leqslant C$, where $C$ is a positive constant depending on $F$, but not on the initial datum $\varphi_{0}$. Thus, we deduce that

$$
\int_{0}^{t}\|\boldsymbol{u}(\tau)\|^{2}+\|\nabla \mu(\tau)\|^{2} \mathrm{~d} \tau \leqslant C, \quad \forall t \geqslant 0
$$


Arguing as in step 2, we take the gradient of $\mu$ and multiply by $\nabla \varphi$. After standard computations, we find

$$
\|\nabla \varphi\| \leqslant C(1+\|\nabla \mu\|) .
$$

Then, aiming to estimate $\bar{\mu}$, we recall the useful inequality (see [20, equations (3.35)-3.37] or [24, proof of theorem 3.15])

$$
\left\|F^{\prime}(\varphi)\right\|_{L^{1}(\Omega)} \leqslant C \int_{\Omega}\left(\varphi-\bar{\varphi}_{0}\right) F^{\prime}(\varphi) \mathrm{d} x+C,
$$

where $C$ depends on $m$. Thus, testing $\mu$ by $\varphi-\bar{\varphi}$, we easily obtain

$$
\left\|F^{\prime}(\varphi)\right\|_{L^{1}(\Omega)} \leqslant C(1+\|\nabla \mu\|)
$$

which, in turn, entails

$$
\|\mu\|_{V} \leqslant C(1+\|\nabla \mu\|) \text {. }
$$

Therefore, (3.5) follows from integrating (3.44)-(3.46) in time on $(t, t+1)$. The proof is complete.

Remark 3.4. A consequence of the proof of theorem 3.1 is the existence of a weak solution to the nonlocal Cahn-Hilliard-Brinkman system with singular potential (please refer to [14] for the local case).

Our next aim is to show the uniqueness of weak solutions. We remind that, in the local case, this is known only in two dimensions (see [27]). To this purpose, we observe that the regularity of the velocity field $\boldsymbol{u} \in L^{2}\left(0, T ; \mathbf{H}_{\sigma}\right)$ is not sufficient. Nonetheless, taking advantage of the equivalent formulation of the Darcy's law (see remark 3.3) and using the global space-time $L^{\infty}$-control of $\varphi$, we prove that the velocity field of any weak solution is indeed more regular.

Lemma 3.1. Let $(\boldsymbol{u}, P, \varphi)$ be a weak solution in the sense of definition 3.1. For any $p \in(1, \infty)$, there exists $C=C(p)>0$, independent of the initial datum, such that

$$
\|\boldsymbol{u}\|_{L^{\infty}\left(0, \infty ; \mathbf{L}^{p}(\Omega)\right)} \leqslant C
$$

Furthermore, there exists $C>0$, independent of the initial datum, such that

$$
\|\boldsymbol{u}\|_{L^{2}(t, t+1 ; \mathbf{V})} \leqslant C, \quad \forall t \geqslant 0 .
$$

Proof. Observe first that, by remark 3.3, up to a redefinition of the pressure, $\boldsymbol{u}$ solves the equation $\boldsymbol{u}=-\nabla P^{*}+(\nabla J * \varphi) \varphi$. Since $\varphi$ is essentially bounded, it is easily seen that

$$
\|(\nabla J * \varphi) \varphi\|_{\mathbf{L}^{\infty}(\Omega \times(0, T))} \leqslant C, \quad \forall T>0 .
$$

Thus, (3.47) follows from (2.5). Let us now apply the curl operator to the Darcy's law. We find (in a distributional sense)

$$
\nabla \times \boldsymbol{u}=-(\nabla J * \varphi) \times \nabla \varphi .
$$

Exploiting once more the $L^{\infty}$-bound of $\varphi$, we reach

$$
\|-(\nabla J * \varphi) \times \nabla \varphi\| \leqslant C\|\nabla \varphi\| .
$$


Then, owing to (3.5), we end up with

$$
\int_{t}^{t+1}\|(\nabla \times \boldsymbol{u})(\tau)\|^{2} \mathrm{~d} \tau \leqslant C .
$$

By virtue of (2.7), the above inequality entails (3.48) and this yields the proof.

We are now in a position to prove the following continuous dependence estimate.

Theorem 3.2. Let $\varphi_{01}$ and $\varphi_{02}$ be two measurable initial data with $F\left(\varphi_{0 i}\right) \in L^{1}(\Omega)$ and $\left|\bar{\varphi}_{0 i}\right|<1, i=1,2$. Then, there exists $C=C\left(T, F\left(\varphi_{01}\right), F\left(\varphi_{02}\right)\right)>0$ such that

$$
\left\|\varphi_{1}(t)-\varphi_{2}(t)\right\|_{V^{\prime}}^{2} \leqslant C\left\|\varphi_{01}-\varphi_{02}\right\|_{V^{\prime}}^{2}+C\left|\bar{\varphi}_{01}-\bar{\varphi}_{02}\right|
$$

for all $0 \leqslant t \leqslant T$. In particular, the weak solution to problem (1.6) and (1.7) is unique.

Proof. Let $\left(\boldsymbol{u}_{1}, P_{1}, \varphi_{1}\right)$ and $\left(\boldsymbol{u}_{2}, P_{2}, \varphi_{2}\right)$ be two weak solutions to problem (1.6) and (1.7) corresponding to $\varphi_{01}$ and $\varphi_{02}$, respectively. Setting $\varphi=\varphi_{1}-\varphi_{2}, \mu=\mu_{1}-\mu_{2}$ and $\boldsymbol{u}=\boldsymbol{u}_{1}-\boldsymbol{u}_{2}$, we have (see remark 3.3)

$$
\begin{aligned}
& (\boldsymbol{u}, \boldsymbol{v})=\left((\nabla J * \varphi) \varphi_{1}, \boldsymbol{v}\right)+\left(\left(\nabla J * \varphi_{2}\right) \varphi, \boldsymbol{v}\right), \quad \forall \boldsymbol{v} \in \mathbf{H}_{\sigma}, \text { a.e. } t \in(0, T), \\
& \left\langle\varphi_{t}, v\right\rangle+(\nabla \mu, \nabla v)=\left(\boldsymbol{u} \varphi_{1}, \nabla v\right)+\left(\boldsymbol{u}_{2} \varphi, \nabla v\right), \quad \forall v \in V, \text { a.e. } t \in(0, T) .
\end{aligned}
$$

Taking $v=1$ in (3.52) we readily obtain that $\bar{\varphi}(t)=\bar{\varphi}(0)$ for all $t \in[0, T]$. Let us now take $v=\mathcal{N}(\varphi-\bar{\varphi})$ in (3.52). By definition of $\mathcal{N}$ and using the chain rule (2.3), we obtain

$$
\frac{1}{2} \frac{\mathrm{d}}{\mathrm{d} t}\|\varphi-\bar{\varphi}\|_{V_{0}^{\prime}}^{2}+(\mu, \varphi-\bar{\varphi})=\mathcal{I}_{1}+\mathcal{I}_{2}
$$

where

$$
\mathcal{I}_{1}=\left(\boldsymbol{u} \varphi_{1}, \nabla \mathcal{N}(\varphi-\bar{\varphi})\right), \quad \mathcal{I}_{2}=\left(\boldsymbol{u}_{2} \varphi, \nabla \mathcal{N}(\varphi-\bar{\varphi})\right)
$$

Thanks to (H.2), we have

$$
\left(F^{\prime}\left(\varphi_{1}\right)-F^{\prime}\left(\varphi_{2}\right), \varphi\right) \geqslant \alpha\|\varphi\|^{2} .
$$

On the other hand, recalling the conservation of the total mass, note that

$$
\left(F^{\prime}\left(\varphi_{1}\right)-F^{\prime}\left(\varphi_{2}\right), \bar{\varphi}\right) \leqslant|\bar{\varphi}(0)|\left(\left\|F^{\prime}\left(\varphi_{1}\right)\right\|_{L^{1}(\Omega)}+\left\|F^{\prime}\left(\varphi_{2}\right)\right\|_{L^{1}(\Omega)}\right) .
$$

Moreover, recalling once more the definition of $\mathcal{N}$, we deduce that

$$
(J * \varphi, \varphi-\bar{\varphi})=(\nabla J * \varphi, \nabla \mathcal{N}(\varphi-\bar{\varphi})) \leqslant \frac{\alpha}{2}\|\varphi\|^{2}+C\|\varphi\|_{*}^{2} .
$$

Therefore, we infer

$$
\frac{1}{2} \frac{\mathrm{d}}{\mathrm{d} t}\|\varphi\|_{*}^{2}+\frac{\alpha}{2}\|\varphi\|^{2} \leqslant \mathcal{I}_{1}+\mathcal{I}_{2}+C\|\varphi\|_{*}^{2}+|\bar{\varphi}(0)| \mathcal{W},
$$


where

$$
\mathcal{W}=C\left(\left\|F^{\prime}\left(\varphi_{1}\right)\right\|_{L^{1}(\Omega)}+\left\|F^{\prime}\left(\varphi_{2}\right)\right\|_{L^{1}(\Omega)}\right) .
$$

Let us proceed to estimate the terms $\mathcal{I}_{i}, i=1,2$. We have

$$
\mathcal{I}_{1} \leqslant\|\boldsymbol{u}\|\|\varphi-\bar{\varphi}\|_{V_{0}^{\prime}}
$$

In order to find a control of $\boldsymbol{u}$ in term of $\varphi$, we take $\boldsymbol{v}=\boldsymbol{u}$ in (3.51) getting

$$
\|\boldsymbol{u}\|^{2}=\left((\nabla J * \varphi) \varphi_{1}, \boldsymbol{u}\right)+\left(\left(\nabla J * \varphi_{2}\right) \varphi, \boldsymbol{u}\right) .
$$

After standard computations, we obtain

$$
\|\boldsymbol{u}\|^{2} \leqslant\left(\left\|\varphi_{1}\right\|_{L^{\infty}(\Omega)}+\left\|\varphi_{2}\right\|_{L^{\infty}(\Omega)}\right)\|\nabla J\|_{\mathbf{L}^{1}(\Omega)}\|\boldsymbol{u}\|\|\varphi\| .
$$

Hence, we arrive at

$$
\|\boldsymbol{u}\| \leqslant C\|\varphi\|
$$

which, in turn, gives

$$
\mathcal{I}_{1} \leqslant \frac{\alpha}{8}\|\varphi\|^{2}+C\|\varphi\|_{*}^{2} .
$$

Regarding $\mathcal{I}_{2}$, by using (2.2), (2.4) and (3.47), we find the control

$$
\begin{aligned}
\mathcal{I}_{2} & \leqslant\left\|\boldsymbol{u}_{2}\right\|_{\mathbf{L}^{6}(\Omega)}\|\varphi\|\|\nabla \mathcal{N}(\varphi-\bar{\varphi})\|_{\mathbf{L}^{3}(\Omega)} \\
& \leqslant C\left\|\boldsymbol{u}_{2}\right\|_{\mathbf{L}^{6}(\Omega)}\|\varphi\|\|\varphi-\bar{\varphi}\|^{\frac{1}{2}}\|\varphi-\bar{\varphi}\|_{V_{0}^{\prime}}^{\frac{1}{2}} \\
& \leqslant \frac{\alpha}{8}\|\varphi\|^{2}+C\|\varphi\|_{*}^{2} .
\end{aligned}
$$

Combining all the previous estimates, we end up with the differential inequality

$$
\frac{1}{2} \frac{\mathrm{d}}{\mathrm{d} t}\|\varphi\|_{*}^{2}+\frac{\alpha}{4}\|\varphi\|^{2} \leqslant C\|\varphi\|_{*}^{2}+|\bar{\varphi}(0)| \mathcal{W}
$$

Therefore, taking into account that $\mathcal{W} \in L^{1}(0, T)$, an application of the Gronwall lemma yields

$$
\left\|\varphi_{1}(t)-\varphi_{2}(t)\right\|_{*}^{2} \leqslant C\left(\left\|\varphi_{01}-\varphi_{02}\right\|_{*}^{2}+\left|\bar{\varphi}_{01}-\bar{\varphi}_{02}\right|\right), \quad \forall t \in[0, T] .
$$

By the equivalence of the norms, (3.50) immediately follows. The proof is complete.

We can also deduce the validity of the energy identity from lemma 3.1. This identity will play a crucial role to study the longtime behavior (see section 6).

Proposition 3.1. Let $\varphi_{0}$ be a measurable function with $F\left(\varphi_{0}\right) \in L^{1}(\Omega)$ and $\left|\bar{\varphi}_{0}\right|<1$. Then, the unique weak solution $(\boldsymbol{u}, P, \varphi)$ to problem (1.6) and (1.7) satisfies the energy identity

$$
\mathcal{E}(\varphi(t))+\int_{s}^{t}\|\boldsymbol{u}(\tau)\|^{2}+\|\nabla \mu(\tau)\|^{2} \mathrm{~d} \tau=\mathcal{E}(\varphi(s)), \quad \forall 0 \leqslant s \leqslant t<\infty .
$$

Proof. For $\kappa>0$, let us introduce the modified energy functional $\mathcal{L}: H \rightarrow \mathbb{R}$ given by

$$
\mathcal{L}(\varphi):=\int_{\Omega} F(\varphi) \mathrm{d} x-\frac{1}{2}(J * \varphi, \varphi)+\frac{\kappa}{2}\|\varphi\|^{2} .
$$


By virtue of (H.1), taking $\kappa>0$ large enough, it is easily seen that $\mathcal{L}$ is proper, convex and lower semicontinuous. On account of the regularity $\varphi \in L^{2}(0, T ; V), \varphi_{t} \in L^{2}\left(0, T ; V^{\prime}\right)$ and $F^{\prime}(\varphi) \in L^{2}(0, T ; V)$, we learn from [13, proposition 4.2] that $\mathcal{L}(\varphi(\cdot))$ is absolutely continuous on $[0, T]$ and

$$
\frac{\mathrm{d}}{\mathrm{d} t} \mathcal{L}(\varphi)=\left\langle F^{\prime}(\varphi)-J * \varphi+\kappa \varphi, \varphi_{t}\right\rangle,=\left\langle\mu, \varphi_{t}\right\rangle+\frac{\kappa}{2} \frac{\mathrm{d}}{\mathrm{d} t}\|\varphi\|^{2},
$$

for almost any $t \in(0, T)$. Here we have also used the chain rule in $L^{2}(0, T ; V) \cap H^{1}\left(0, T ; V^{\prime}\right)$. Taking $v=\mu$ in (3.1) and summing up to the above equality, we find

$$
\frac{\mathrm{d}}{\mathrm{d} t} \mathcal{E}(\varphi)-(\boldsymbol{u} \varphi, \nabla \mu)+\|\nabla \mu\|^{2}=0
$$

Then, testing the Darcy's law by $\boldsymbol{u}$, we have

$$
\|\boldsymbol{u}\|^{2}=(\mu \nabla \varphi, \boldsymbol{u}) .
$$

Note that the above test by $\boldsymbol{u}$ is well defined due to lemma 3.1 (see, in particular, (3.47)). By the classical result on the product rule in Sobolev spaces, we can rewrite (3.54) as

$$
\|\boldsymbol{u}\|^{2}=-(\varphi \nabla \mu, \boldsymbol{u}) .
$$

Thus, collecting the above equalities, we end up with

$$
\frac{\mathrm{d}}{\mathrm{d} t} \mathcal{E}(\varphi)+\|\boldsymbol{u}\|^{2}+\|\nabla \mu\|^{2}=0
$$

An integration on $(s, t), 0 \leqslant s<t$, entails the energy identity (3.53).

\section{Strong solutions and regularity properties}

In this section we prove the global existence of the (unique) strong solution to problem (1.6) and (1.7) under a natural assumption on the initial datum. This will be achieved via a priori higher-order estimates. As mentioned in the introduction, this result (together with the uniqueness of weak solutions) in the three dimensional case, points out the gap between the local and the nonlocal versions of the Cahn-Hilliard-Hele-Shaw model with logarithmic potential. In the former case, we remind that the existence of a global-in-time strong solution has been proven only for initial data close to local minimizers of the Ginzburg-Landau functional. On account of the parabolic dissipative nature of the system, we also show the time regularization of weak solutions.

Theorem 4.1. Let $\varphi_{0}$ be a measurable function with $F\left(\varphi_{0}\right) \in L^{1}(\Omega),\left|\bar{\varphi}_{0}\right|<1$ and $\nabla F^{\prime}\left(\varphi_{0}\right) \in \mathbf{H}$. Then, the weak solution is a strong solution to problem (1.6) and (1.7) on $[0, T]$ and satisfies

$$
\begin{aligned}
& \boldsymbol{u} \in L^{\infty}(0, T ; \mathbf{V}) \cap L^{\frac{8}{d}}\left(0, T ; \mathbf{W}^{1,4}(\Omega)\right) \cap L^{2}\left(0, T ; \mathbf{W}^{1, p}(\Omega)\right), \\
& P \in L^{\infty}\left(0, T ; W^{1, \frac{3}{2}}(\Omega)\right) \cap L^{2}(0, T ; V), \\
& \varphi \in L^{\infty}(0, T ; V) \cap L^{\frac{8}{d}}\left(0, T ; W^{1,4}(\Omega)\right) \cap L^{2}\left(0, T ; W^{1, p}(\Omega)\right), \\
& \varphi_{t} \in L^{\infty}\left(0, T ; V^{\prime}\right) \cap L^{2}(0, T ; H) \\
& F^{\prime}(\varphi) \in L^{\infty}(0, T ; V) \\
& \mu \in L^{\infty}(0, T ; V) \cap L^{\frac{8}{d}}\left(0, T ; W^{1,4}(\Omega)\right) \cap L^{2}\left(0, T ; H^{2}(\Omega)\right),
\end{aligned}
$$

for any $4<p<\infty$ if $d=2$ or $4<p \leqslant 6$ if $d=3$. 
Proof. Let $(\boldsymbol{u}, P, \varphi)$ be the unique global-in-time weak solution to problem (1.6) and (1.7). Thanks to the additional assumption on the initial datum, we aim to show higher-order regularity properties. We divide the proof into three steps.

\subsection{Smoothing effect on time derivatives}

For any $h>0$, let us introduce the difference quotient $D^{h} u=\frac{1}{h}(u(t+h)-u(t))$. We first consider the Darcy's equation at two different times $t$ and $t+h$. By subtracting them, it is evident that (see remark 3.3)

$$
\left(D^{h} \boldsymbol{u}, \boldsymbol{v}\right)=\left(\left(\nabla J * D^{h} \varphi\right) \varphi(\cdot+h), \boldsymbol{v}\right)+\left((\nabla J * \varphi) D^{h} \varphi, \boldsymbol{v}\right),
$$

for all $\boldsymbol{v} \in \mathbf{H}_{\sigma}$ and for almost any $t>0$. Choosing $\boldsymbol{v}=D^{h} \boldsymbol{u}$, we deduce that

$$
\left\|D^{h} \boldsymbol{u}\right\|^{2}=\left(\left(\nabla J * D^{h} \varphi\right) \varphi(\cdot+h), D^{h} \boldsymbol{u}\right)+\left((\nabla J * \varphi) D^{h} \varphi, D^{h} \boldsymbol{u}\right) .
$$

By (H.1) and (3.4), we have

$$
\begin{aligned}
& \left(\left(\nabla J * D^{h} \varphi\right) \varphi(\cdot+h), D^{h} \boldsymbol{u}\right)+\left((\nabla J * \varphi) D^{h} \varphi, D^{h} \boldsymbol{u}\right) \\
& \leqslant\left(\|\varphi(\cdot+h)\|_{L^{\infty}(\Omega)}\|\nabla J\|_{\mathbf{L}^{1}(\Omega)}+\|\nabla J * \varphi\|_{\mathbf{L}^{\infty}(\Omega)}\right)\left\|D^{h} \varphi\right\|\left\|D^{h} \boldsymbol{u}\right\| \\
& \quad \leqslant C\left\|D^{h} \varphi\right\|\left\|D^{h} \boldsymbol{u}\right\|,
\end{aligned}
$$

which, in turn, gives

$$
\left\|D^{h} \boldsymbol{u}\right\| \leqslant C\left\|D^{h} \varphi\right\| .
$$

Subtracting now the weak formulation (3.1) evaluated at time $t$ from the one at time $t+h$ we get

$$
\left\langle\partial_{t} D^{h} \varphi, v\right\rangle+\left(\nabla D^{h} \mu, \nabla v\right)=\left(\varphi(\cdot+h) D^{h} \boldsymbol{u}, \nabla v\right)+\left(\boldsymbol{u} D^{h} \varphi, \nabla v\right)
$$

for every $v \in V$ and almost any $t \in(0, T)$. Taking $v=\mathcal{N} D^{h} \varphi$, we obtain

$$
\frac{1}{2} \frac{\mathrm{d}}{\mathrm{d} t}\left\|D^{h} \varphi\right\|_{V_{0}^{\prime}}^{2}+\left(D^{h} \mu, D^{h} \varphi\right)=\mathcal{J}_{1}+\mathcal{J}_{2}
$$

where

$$
\mathcal{J}_{1}=\left(\varphi(\cdot+h) D^{h} \boldsymbol{u}, \nabla \mathcal{N} D^{h} \varphi\right), \quad \mathcal{J}_{2}=\left(\boldsymbol{u} D^{h} \varphi, \nabla \mathcal{N} D^{h} \varphi\right)
$$

By virtue of (H.1) and (H.2), we have

$$
\frac{1}{h}\left(F^{\prime}(\varphi(\cdot+h))-F^{\prime}(\varphi), D^{h} \varphi\right) \geqslant \alpha\left\|D^{h} \varphi\right\|^{2}
$$

and

$$
\left(J * D^{h} \varphi, D^{h} \varphi\right)=\left(\nabla J * D^{h} \varphi, \nabla \mathcal{N} D^{h} \varphi\right) \leqslant \frac{\alpha}{6}\left\|D^{h} \varphi\right\|^{2}+C\left\|D^{h} \varphi\right\|_{V_{0}^{\prime}}^{2}
$$

Let us control the terms $\mathcal{J}_{i}, i=1,2$. By (2.4), (2.2), (3.47) and (4.1), we find

$$
\mathcal{J}_{1} \leqslant C\left\|D^{h} \boldsymbol{u}\right\|\left\|D^{h} \varphi\right\|_{V_{0}^{\prime}} \leqslant \frac{\alpha}{6}\left\|D^{h} \varphi\right\|^{2}+C\left\|D^{h} \varphi\right\|_{*}^{2},
$$


and

$\mathcal{J}_{2} \leqslant\|\boldsymbol{u}\|_{\mathbf{L}^{6}(\Omega)}\left\|D^{h} \varphi\right\|\left\|\nabla \mathcal{N} D^{h} \varphi\right\|_{\mathbf{L}^{3}(\Omega)} \leqslant C\left\|D^{h} \varphi\right\|^{\frac{3}{2}}\left\|D^{h} \varphi\right\|_{V_{0}^{\prime}}^{\frac{1}{2}} \leqslant \frac{\alpha}{6}\left\|D^{h} \varphi\right\|^{2}+C\left\|D^{h} \varphi\right\|_{*}^{2}$.

Therefore, collecting the above estimates, we arrive at the differential inequality

$$
\frac{1}{2} \frac{\mathrm{d}}{\mathrm{d} t}\left\|D^{h} \varphi\right\|_{*}^{2}+\frac{\alpha}{2}\left\|D^{h} \varphi\right\|^{2} \leqslant C\left\|D^{h} \varphi\right\|_{*}^{2} .
$$

Thus an application of the Gronwall lemma yields

$$
\left\|D^{h} \varphi(t)\right\|_{*}^{2} \leqslant\left\|D^{h} \varphi(0)\right\|_{*}^{2} e^{C T}, \quad \forall t \in[0, T] .
$$

At this point, to deduce a global-in-time estimate we need to find a bound for $\left\|D^{h} \varphi(0)\right\|_{*}$. Taking advantage of (H.2), and using (3.47), we observe that

$$
\begin{aligned}
\frac{1}{2} \frac{\mathrm{d}}{\mathrm{d} t}\left\|\varphi-\varphi_{0}\right\|_{V_{0}^{\prime}}^{2} & =\left\langle\varphi_{t}, \mathcal{N}\left(\varphi-\varphi_{0}\right)\right\rangle=\left(\mu, \varphi-\varphi_{0}\right)+\left(\boldsymbol{u} \varphi, \nabla \mathcal{N}\left(\varphi-\varphi_{0}\right)\right) \\
& =-\left(F^{\prime}(\varphi), \varphi-\varphi_{0}\right)+\left(J * \varphi, \varphi-\varphi_{0}\right)+\left(\boldsymbol{u} \varphi, \nabla \mathcal{N}\left(\varphi-\varphi_{0}\right)\right) \\
& \leqslant-\left(\nabla F^{\prime}\left(\varphi_{0}\right), \nabla \mathcal{N}\left(\varphi-\varphi_{0}\right)\right)+\left(\nabla J * \varphi, \nabla \mathcal{N}\left(\varphi-\varphi_{0}\right)\right)+\left(\boldsymbol{u} \varphi, \nabla \mathcal{N}\left(\varphi-\varphi_{0}\right)\right) \\
& \leqslant\left(C+\left\|\nabla F^{\prime}\left(\varphi_{0}\right)\right\|\right)\left\|\varphi-\varphi_{0}\right\|_{V_{0}^{\prime}},
\end{aligned}
$$

for almost every $t \geqslant 0$. An integration in time leads to

$$
\frac{1}{2}\left\|\varphi(t)-\varphi_{0}\right\|_{V_{0}^{\prime}}^{2} \leqslant\left(C+\left\|\nabla F^{\prime}\left(\varphi_{0}\right)\right\|\right) \int_{0}^{t}\left\|\varphi(\tau)-\varphi_{0}\right\|_{V_{0}^{\prime}} \mathrm{d} \tau, \quad \forall t \geqslant 0 .
$$

Accordingly, an application of the Gronwall lemma (see [7, lemma A.5]) implies

$$
\left\|\varphi(t)-\varphi_{0}\right\|_{V_{0}^{\prime}} \leqslant\left(C+\left\|\nabla F^{\prime}\left(\varphi_{0}\right)\right\|\right) t, \quad \forall t \geqslant 0 .
$$

Taking $t=h$, it follows that

$$
\left\|D^{h} \varphi(0)\right\|_{*} \leqslant\left(C+\left\|\nabla F^{\prime}\left(\varphi_{0}\right)\right\|\right), \quad \forall h>0 .
$$

Combining (4.5) with (4.6), we end up with

$$
\left\|D^{h} \varphi(t)\right\|_{*} \leqslant C, \quad \forall t \in[0, T],
$$

where $C$ depends on $\varphi_{0}$ and $T$. Owing to (4.4), we also infer that

$$
\left\|D^{h} \varphi\right\|_{L^{2}(0, T ; H)} \leqslant C .
$$

Since $\varphi \in H^{1}\left(0, T ; V^{\prime}\right)$, we recall that $D^{h} \varphi$ converges to $\varphi_{t}$ strongly in $L^{2}\left(0, T ; V^{\prime}\right)$ as $h \rightarrow 0$ (see [7, proposition A.6]). Then, by the uniqueness of the weak limit, we find the controls

$$
\left\|\varphi_{t}\right\|_{L^{\infty}\left(0, T ; V^{\prime}\right)}+\left\|\varphi_{t}\right\|_{L^{2}(0, T ; H)} \leqslant C .
$$

Besides, by (4.1), (4.7) and [7, corollary A.2 and proposition A.7], we deduce that

$$
\left\|\boldsymbol{u}_{t}\right\|_{L^{2}\left(0, T ; \mathbf{H}_{\sigma}\right)} \leqslant C .
$$

\section{2. $\quad L^{\infty}$-in time uniform estimates}

Our next concern is to establish global-in-time bounds on $\boldsymbol{u}, P$ and $\varphi$. By (3.4), (3.46), (3.47), and (4.8), it is easily seen that 


$$
\|\mu\|_{L^{\infty}(0, T ; V)} \leqslant C .
$$

Then, by virtue of (3.45), we get

$$
\|\varphi\|_{L^{\infty}(0, T ; V)} \leqslant C \text {. }
$$

In light of the latter bound, by using (2.7) and (3.49), we have

$$
\|\boldsymbol{u}\|_{\mathbf{v}} \leqslant C\left(1+\|\varphi\|_{L^{\infty}(\Omega)}\|\nabla \varphi\|\right),
$$

which, in turn, entails

$$
\|\boldsymbol{u}\|_{L^{\infty}(0, T ; \mathbf{V})} \leqslant C .
$$

As a consequence, we obtain

$$
\|P\|_{L^{\infty}\left(0, T ; W^{1, \frac{3}{2}}(\Omega)\right)} \leqslant C .
$$

\subsection{Higher-order estimates in space}

We proceed by proving higher-order regularity with respect to the space variables. In this regard, by (3.4) and (3.47), we observe that

$$
\|\boldsymbol{u} \varphi\|_{L^{\infty}\left(0, T ; \mathbf{L}^{p}(\Omega)\right)} \leqslant C,
$$

for any $p \geqslant 2$, where $C$ depends on $p$. Now, we read (3.1) as the Neumann problem for $\mu$. Then, the weak $L^{p}$-regularity (see [39]) entails that, for any $p>1$, there exists $C=C(p)>0$ such that

$$
\|\mu\|_{W^{1, p}(\Omega)} \leqslant C\left(\left\|\varphi_{t}\right\|_{\left(W^{1, p^{\prime}}(\Omega)\right)^{\prime}}+\|\boldsymbol{u} \cdot \nabla \varphi\|_{\left(W^{1, p^{\prime}}(\Omega)\right)^{\prime}}+\|\mu\|_{\left(W^{1, p^{\prime}}(\Omega)\right)^{\prime}}\right),
$$

where $\frac{1}{p}+\frac{1}{p^{\prime}}=1$. In light of (4.10) and (4.13), we have

$$
\|\mu\|_{W^{1, p}(\Omega)} \leqslant C\left(\left\|\varphi_{t}\right\|+\|\boldsymbol{u} \varphi\|_{\mathbf{L}^{p}(\Omega)}+\|\mu\|\right) \leqslant C\left(1+\left\|\varphi_{t}\right\|\right),
$$

for any $1<p<\infty$ if $d=2$ or $1<p \leqslant 6$ if $d=3$. This gives

$$
\|\mu\|_{L^{2}\left(0, T ; W^{1, p}(\Omega)\right)} \leqslant C \text {. }
$$

To recover further integrability on $\varphi$, we argue as in [24, proposition 4.2] (see also [21]). We first deduce that $F^{\prime \prime}(\varphi) \nabla \varphi \in L^{2}\left(0, T ; \mathbf{L}^{p}(\Omega)\right)$, for any $p \geqslant 1$. Then, exploiting (H.2), we reach

$$
\|\nabla \varphi\|_{\mathbf{L}^{p}(\Omega)} \leqslant C\left(1+\|\nabla \mu\|_{\mathbf{L}^{p}(\Omega)}\right),
$$

which, in turn, entails

$$
\|\varphi\|_{L^{2}\left(0, T ; W^{1, p}(\Omega)\right)} \leqslant C .
$$

Accordingly, we can improve the integrability of the convective term $\boldsymbol{u} \cdot \nabla \varphi$. By (3.47) and (4.16), we find

$$
\|\boldsymbol{u} \cdot \nabla \varphi\|_{L^{2}(0, T ; H)} \leqslant C .
$$

Due to this, the regularity theory of the Neumann problem yields

$$
\|\mu\|_{L^{2}\left(0, T ; H^{2}(\Omega)\right)} \leqslant C .
$$


By (2.1) and the above estimates, we also learn that

$$
\|\nabla \mu\|_{L^{\frac{8}{a}}\left(0, T ; \mathbf{L}^{4}(\Omega)\right)}+\|\nabla \varphi\|_{L^{\frac{8}{d}\left(0, T ; \mathbf{L}^{4}(\Omega)\right)}} \leqslant C .
$$

Finally, we consider again (3.49). It follows that, for any $1<p<\infty$ if $d=2$ or $1<p \leqslant 6$ if $d=3$, there exists $C=C(p)>0$ such that

$$
\|\nabla \times \boldsymbol{u}\|_{L^{\frac{8}{d}}\left(0, T ; \mathbf{L}^{4}(\Omega)\right)}+\|\nabla \times \boldsymbol{u}\|_{L^{2}\left(0, T ; \mathbf{L}^{p}(\Omega)\right)} \leqslant C .
$$

On account of [28, theorem 3.5], there exists $\mathbf{g}$ such that $\nabla \times \mathbf{g}=\boldsymbol{u}$ and $-\Delta \mathbf{g}=\nabla \times \boldsymbol{u}$ in $\Omega$, with $\mathbf{g} \cdot \boldsymbol{n}=0$ on $\partial \Omega$. By the regularity theory for the Neumann problem, we obtain

$$
\|\mathbf{g}\|_{L^{\frac{8}{d}\left(0, T ; \mathbf{W}^{2,4}(\Omega)\right)}}+\|\mathbf{g}\|_{L^{2}\left(0, T ; \mathbf{W}^{2, p}(\Omega)\right)} \leqslant C .
$$

Thus, we infer that

$$
\|\boldsymbol{u}\|_{L^{\frac{8}{d}}\left(0, T ; \mathbf{W}^{1,4}(\Omega)\right)}+\|\boldsymbol{u}\|_{L^{2}\left(0, T ; \mathbf{W}^{1, p}(\Omega)\right)} \leqslant C .
$$

The further regularity of the pressure easily follows from (2.6) and the above estimates. This concludes the proof.

We are now in a position to state that any weak solution becomes instantaneously a strong solution. To this aim, we consider a generic weak solution $(\boldsymbol{u}, P, \varphi)$ departing from a measurable initial datum $\varphi_{0}$ such that $\left|\bar{\varphi}_{0}\right| \leqslant m$, for a fixed $m \in[0,1)$. Accordingly, throughout this section, the generic positive constant $C$ may depend on $m$, but will be independent of the initial datum.

Theorem 4.2. Let $(\boldsymbol{u}, P, \varphi)$ be a weak solution to problem (1.6) and (1.7). For any $\sigma>0$, there exists $C=C(\sigma)>0$ such that

$$
\begin{aligned}
& \left\|\varphi_{t}\right\|_{L^{\infty}\left(\sigma, \infty ; V^{\prime}\right)}+\|\mu\|_{L^{\infty}(\sigma, \infty ; V)} \leqslant C, \\
& \sup _{t \geqslant \sigma}\|\boldsymbol{u}(t)\|_{\mathbf{v}}+\sup _{t \geqslant \sigma}\|\varphi(t)\|_{V} \leqslant C,
\end{aligned}
$$

$\left\|\boldsymbol{u}_{t}\right\|_{L^{2}\left(t, t+1 ; \mathbf{H}_{\sigma}\right)}+\left\|\varphi_{t}\right\|_{L^{2}(t, t+1 ; H)}+\|\mu\|_{L^{2}\left(t, t+1 ; H^{2}(\Omega)\right)}+\|\nabla \varphi\|_{L^{\frac{8}{d}\left(0, T ; \mathbf{L}^{4}(\Omega)\right)}} \leqslant C, \quad \forall t \geqslant \sigma$.

Moreover, for any $4<p<\infty$ if $d=2$ or $4<p \leqslant 6$ if $d=3$ and $\sigma>0$, there exists $C=C(\sigma, p)$ such that

$$
\|\boldsymbol{u}\|_{L^{2}\left(t, t+1 ; \mathbf{W}^{1, p}(\Omega)\right)}+\|\varphi\|_{L^{2}\left(t, t+1 ; W^{1, p}(\Omega)\right)}+\|\mu\|_{L^{2}\left(t, t+1 ; W^{1, p}(\Omega)\right)} \leqslant C, \quad \forall t \geqslant \sigma .
$$

Proof. The proof of theorem 4.2 can be obtained by arguing as in theorem 4.1. We recall the differential inequality

$$
\frac{1}{2} \frac{\mathrm{d}}{\mathrm{d} t}\left\|D^{h} \varphi\right\|_{*}^{2}+\frac{\alpha}{2}\left\|D^{h} \varphi\right\|^{2} \leqslant C\left\|D^{h} \varphi\right\|_{*}^{2}
$$

Observing that, for any $0<h<1$,

$$
\left\|D^{h} \varphi\right\|_{L^{2}\left(t, t+1 ; V^{\prime}\right)} \leqslant\left\|\varphi_{t}\right\|_{L^{2}\left(t, t+2 ; V^{\prime}\right)}, \quad \forall t \geqslant 0,
$$

and, by comparison,

$$
\left\|\varphi_{t}\right\|_{V^{\prime}} \leqslant C\left(\|\nabla \mu\|+\|\boldsymbol{u}\|_{\mathbf{L}^{3}(\Omega)}\|\varphi\|_{L^{6}(\Omega)}\right),
$$

we deduce from (3.5) and (3.47) that 


$$
\left\|D^{h} \varphi\right\|_{L^{2}\left(t, t+1 ; V^{\prime}\right)} \leqslant C, \quad \forall t \geqslant 0,
$$

where $C$ is independent of $h$. Applying the uniform Gronwall lemma (see e.g. [44, lemma 1.1, chapter III]), for every $\sigma>0$ there exists $C=C(\sigma)>0$ such that

$$
\left\|D^{h} \varphi\right\|_{*}^{2} \leqslant C, \quad \forall t \geqslant \sigma .
$$

Since $D^{h} \varphi$ converges to $\varphi_{t}$ strongly in $L^{2}\left(0, T ; V^{\prime}\right)$ as $h \rightarrow 0$, for any $T>0$, we conclude that

$$
\left\|\varphi_{t}\right\|_{L^{\infty}\left(\sigma, \infty ; V^{\prime}\right)} \leqslant C \text {. }
$$

Furthermore, an integration of (4.17) on the time interval $(t, t+1)$, with $t \geqslant \sigma$, together with (4.1), yields also

$$
\left\|D^{h} \varphi\right\|_{L^{2}(t, t+1 ; H)}+\left\|D^{h} \boldsymbol{u}\right\|_{L^{2}\left(t, t+1 ; \mathbf{H}_{\sigma}\right)} \leqslant C, \quad \forall t \geqslant \sigma .
$$

A passage to the limit as $h \rightarrow 0$ in the above estimate leads to

$$
\left\|\varphi_{t}\right\|_{L^{2}(t, t+1 ; H)}+\left\|\boldsymbol{u}_{t}\right\|_{L^{2}\left(t, t+1 ; \mathbf{H}_{\sigma}\right)} \leqslant C, \quad \forall t \geqslant \sigma .
$$

All the remaining estimates follow by repeating line by line the arguments employed in the steps 2 and 3 of the proof of theorem 4.1.

\section{5. $d=2$ : separation property and its consequences}

In this section we address the physically relevant quantitative property concerning the instantaneous and uniform-in-time separation from the pure phases. In other words, in the two dimensional case we can prove that the concentration parameter stays away from the singular values of the potential. Consequently, being the potential and its derivative globally bounded in $L^{\infty}$-norm, we are also able to show a further regularization property. More precisely, we have

Theorem 5.1. Let $d=2$. Assume that $F \in \mathcal{C}^{3}(-1,1)$ satisfies the following conditions

$$
F^{\prime \prime}(s) \leqslant e^{C\left|F^{\prime}(s)\right|+C}, \quad F^{\prime}(s) F^{\prime \prime \prime}(s) \geqslant 0, \quad\left|F^{\prime \prime \prime}(s)\right| \leqslant C F^{\prime \prime}(s)^{2}, \quad \forall s \in(-1,1),
$$

for some positive constant $C$. Then, we have:

(i) If $(\boldsymbol{u}, P, \varphi)$ is a strong solution to problem (1.6) and (1.7) provided by theorem 4.1 and $\left\|\varphi_{0}\right\|_{L^{\infty}(\Omega)} \leqslant 1-\delta_{0}$, for some $\delta_{0} \in(0,1]$, then there exists $\delta \in(0,1]$ depending on the initial datum such that

$$
\sup _{t \geqslant 0}\|\varphi(t)\|_{L^{\infty}(\Omega)} \leqslant 1-\delta .
$$

(ii) If $(\boldsymbol{u}, P, \varphi)$ is a weak solution to problem (1.6) and (1.7) provided by theorem 3.1 then, for any $\sigma>0$, there exists $\delta=\delta(\sigma, m) \in(0,1]$ such that

$$
\sup _{t \geqslant \sigma}\|\varphi(t)\|_{L^{\infty}(\Omega)} \leqslant 1-\delta .
$$

Proof. Let $(\boldsymbol{u}, P, \varphi)$ be the strong solution to problem (1.6) and (1.7) provided by theorem 4.1. In light of the regularity $F^{\prime}(\varphi) \in L^{\infty}(0, \infty ; V)$ and the additional assumptions 
on $F$, arguing as in [24, theorem 5.2], we deduce that $F^{\prime \prime}(\varphi) \in L^{\infty}\left(0, \infty ; L^{p}(\Omega)\right)$ and $F^{\prime \prime \prime}(\varphi) \in L^{\infty}\left(0, \infty ; L^{p}(\Omega)\right)$, for any $2 \leqslant p<\infty$. Then, repeating line by line the proofs of [24, theorems 5.2 and 6.7], we arrive at the differential inequality, for any $k \in \mathbb{N}$,

$$
\frac{\mathrm{d}}{\mathrm{d} t}\left\|F^{\prime}(\varphi)\right\|_{L^{2^{k}}(\Omega)}^{2^{k}}+\left\|F^{\prime}(\varphi)\right\|_{L^{2^{k}}(\Omega)}^{2^{k}} \leqslant C 2^{6 k}\left(1+\left\|F^{\prime}(\varphi)\right\|_{L^{2 k-1}}^{2^{k}}\right)
$$

for almost every $t \geqslant 0$, and for some positive constant $C$ depending on the initial datum, but independent of $k$. Applying the Gronwall lemma, we find

$$
\begin{aligned}
\max _{t \geqslant 0} \int_{\Omega}\left|F^{\prime}(\varphi(t))\right|^{2^{k}} \mathrm{~d} x \leqslant & \left\|F^{\prime}(\varphi(0))\right\|_{L^{2^{k}(\Omega)}}^{\mathrm{e}^{-t}} \\
& +C 2^{6 k}\left(1+\max _{t \geqslant 0}\left[\int_{\Omega}\left|F^{\prime}(\varphi(t))\right|^{2^{k-1}} \mathrm{~d} x\right]^{2}\right)\left(1-\mathrm{e}^{-t}\right) .
\end{aligned}
$$

Let us introduce

$$
\Lambda=\max \left\{1,\left\|F^{\prime}\left(\varphi_{0}\right)\right\|_{L^{\infty}(\Omega)}, \max _{t \geqslant 0} \int_{\Omega}\left|F^{\prime}(\varphi(t))\right| \mathrm{d} x\right\},
$$

which is finite on account of the assumption on the initial datum. We rewrite the inequality (5.3) as follows

$$
\begin{aligned}
\max _{t \geqslant 0} \int_{\Omega}\left|F^{\prime}(\varphi(t))\right|^{2^{k}} \mathrm{~d} x & \leqslant \max \left\{C \Lambda^{2^{k}}, C 2^{6 k} \Lambda+C 2^{6 k}\left(\max _{t \geqslant 0} \int_{\Omega}\left|F^{\prime}(\varphi(t))\right|^{2^{k-1}} \mathrm{~d} x\right)^{2}\right\} \\
& \leqslant C 2^{6 k+1} \max \left\{\Lambda^{2^{k}},\left(\max _{t \geqslant 0} \int_{\Omega}\left|F^{\prime}(\varphi(t))\right|^{2^{k-1}} \mathrm{~d} x\right)^{2}\right\},
\end{aligned}
$$

for some $C$ independent of $k$. Thus, setting

$$
Q_{k}=\max _{t \geqslant 0} \int_{\Omega}\left|F^{\prime}(\varphi(t))\right|^{2^{k}} \mathrm{~d} x
$$

we deduce the iterative inequality for any $k \in \mathbb{N}$

$$
Q_{k} \leqslant C 2^{6 k}\left(\Lambda^{2^{k}}+Q_{k-1}^{2}\right), \quad Q_{0} \leqslant \Lambda
$$

Then, an iteration argument yields

$$
Q_{k} \leqslant C^{K_{1} 2^{k}} 2^{K_{2} 2^{k}} \Lambda^{2^{k}}
$$

where $K_{1}=\sum_{m=0}^{\infty} \frac{1}{2^{m}}$ and $K_{2}=\sum_{m=0}^{k} \frac{6 m+1}{2^{m}}$. Taking now the limit $k \rightarrow \infty$ of the $\frac{1}{2^{k}}$ power of both sides of (5.4), we obtain

$$
\max _{t \geqslant 0}\left\|F^{\prime}(\varphi(t))\right\|_{L^{\infty}(\Omega)} \leqslant C \Lambda,
$$

for some positive constant $C$ which depends on the initial datum. Thanks to (H.2), the above inequality implies (5.1). The proof of (5.2) can be carried out arguing in the same way (see[24, theorem 5.2] for details).

Remark 5.1. We note that the additional assumptions on the singular potential $F$ of theorem 5.1 are satisfied by the physically relevant logarithmic potential (1.5). 
Remark 5.2. Property (i) essentially says that if the initial datum is strictly separated from the pure phases then the corresponding solution remains separated for any time $t>0$. This implies that the solution to problem (1.6) and (1.7) is also a solution to the same problem where the singular potential $F$ is replaced by a regular extension $F_{R}$ (on $\mathbb{R}$ ) of polynomial growth outside the interval $[-1+\delta, 1-\delta]$. In other words, there exist solutions to problem (1.6) and (1.7) with a regular potential $F_{R}$, whose ranges are in $[-1,1]$ (i.e. the physical range). We recall that this property is often observed in numerical simulations for Cahn-Hilliard type equations. On the other hand, from the theoretical viewpoint, only the global boundedness is known for solutions to problem (1.6) and (1.7) with $F_{R}$ (see [17]).

Taking advantage of the above result we can prove further regularization properties. In order to enhance the spatial Sobolev regularity of the solution $\varphi$ up to $H^{2}(\Omega)$, we need a slightly higher regularity of the interaction kernel $J$, namely

$$
J \in W^{2,1}\left(\mathcal{B}_{\delta}\right) \text {, where } \mathcal{B}_{\delta}=\left\{x \in \mathbb{R}^{d}:|x|<\delta\right\} \text { with } \delta \sim \operatorname{diam}(\Omega) \text { such that } \bar{\Omega} \subset \mathcal{B}_{\delta}
$$
or $J$ is an admissible kernel in the sense of [5, definition 1].

We notice that both Newtonian and Bessel potentials fulfill the second part of (H.3).

Let us fix $m \in[0,1)$ and consider a weak solution $(\boldsymbol{u}, P, \varphi)$ such that $\left|\bar{\varphi}_{0}\right| \leqslant m$. In particular, we remind once more that the generic positive constant $C$ may depend on $m$, but will be independent of the initial datum.

Theorem 5.2. Let $d=2$ and let the assumptions of theorem 5.1 hold. Then, for any $\sigma>0$, there exists $C=C(\sigma)>0$ such that

$$
\left\|\boldsymbol{u}_{t}\right\|_{L^{\infty}\left(\sigma, \infty ; \mathbf{H}_{\sigma}\right)}+\left\|\varphi_{t}\right\|_{L^{\infty}(\sigma, \infty ; H)} \leqslant C
$$

and, for any $1 \leqslant p<\infty$, there exists $C=C(\sigma, p)>0$ such that

$$
\sup _{t \geqslant \sigma}\|\mu(t)\|_{H^{2}(\Omega)}+\sup _{t \geqslant \sigma}\|\varphi(t)\|_{W^{1, p}(\Omega)} \leqslant C .
$$

In addition, if $J$ satisfies the assumption (H.3), there exists a constant $C=C(\sigma)>0$ such that

$$
\sup _{t \geqslant \sigma}\|\boldsymbol{u}(t)\|_{\mathbf{H}^{2}(\Omega)}+\sup _{t \geqslant \sigma}\|\varphi(t)\|_{H^{2}(\Omega)} \leqslant C .
$$

Proof. Given $\sigma>0$, we recall that any weak solution is a strong solution on the time interval $\left(\frac{\sigma}{2}, \infty\right)$ and satisfies the estimates provided by theorem 4.2. Let us test (4.2) by $D^{h} \varphi$. We have

$$
\frac{1}{2} \frac{\mathrm{d}}{\mathrm{d} t}\left\|D^{h} \varphi\right\|^{2}+\left(\nabla D^{h} \mu, \nabla D^{h} \varphi\right)=\mathcal{H}_{1}+\mathcal{H}_{2}
$$

where

$$
\mathcal{H}_{1}=\left(\varphi(\cdot+h) D^{h} \boldsymbol{u}, \nabla D^{h} \varphi\right), \quad \mathcal{H}_{2}=\left(\boldsymbol{u} D^{h} \varphi, \nabla D^{h} \varphi\right) .
$$

By (H.2), we first notice that

$$
\left(\nabla D^{h} \mu, \nabla D^{h} \varphi\right) \geqslant \alpha\left\|\nabla D^{h} \varphi\right\|^{2}-\left|\left(\nabla J * D^{h} \varphi, \nabla D^{h} \varphi\right)\right|-\left|\left(\nabla \varphi D^{h} F^{\prime \prime}(\varphi), \nabla D^{h} \varphi\right)\right| .
$$

It is evident that

$$
\left|\left(\nabla J * D^{h} \varphi, \nabla D^{h} \varphi\right)\right| \leqslant \frac{\alpha}{8}\left\|\nabla D^{h} \varphi\right\|^{2}+C\left\|D^{h} \varphi\right\|^{2} .
$$


On the other hand, recalling that $D^{h} F^{\prime \prime}(\varphi)=D^{h} \varphi \int_{0}^{1} F^{\prime \prime \prime}(s \varphi(t+h)+(1-s) \varphi(t)) \mathrm{d} s$, from the assumptions on $F$ and exploiting (2.1) and theorem 5.1, we infer that

$$
\begin{aligned}
\left|\left(\nabla \varphi D^{h} F^{\prime \prime}(\varphi), \nabla D^{h} \varphi\right)\right| & \leqslant C\|\nabla \varphi\|_{\mathbf{L}^{4}(\Omega)}\left\|D^{h} \varphi\right\|_{L^{4}(\Omega)}\left\|\nabla D^{h} \varphi\right\| \\
& \leqslant \frac{\alpha}{8}\left\|\nabla D^{h} \varphi\right\|^{2}+C\|\nabla \varphi\|_{\mathbf{L}^{4}(\Omega)}^{4}\left\|D^{h} \varphi\right\|^{2} .
\end{aligned}
$$

We proceed by estimating the two terms $\mathcal{H}_{i}, i=1,2$, on the right-hand side. Owing to (3.4), (3.47) and (4.1), we obtain

$$
\mathcal{H}_{1} \leqslant C\left\|D^{h} \varphi\right\|\left\|\nabla D^{h} \varphi\right\| \leqslant C\left\|D^{h} \varphi\right\|^{2}+\frac{\alpha}{8}\left\|\nabla D^{h} \varphi\right\|^{2},
$$

and

$$
\mathcal{H}_{2} \leqslant C\|\boldsymbol{u}\|_{\mathbf{L}^{4}(\Omega)}\left\|D^{h} \varphi\right\|_{L^{4}(\Omega)}\left\|\nabla D^{h} \varphi\right\| \leqslant \frac{\alpha}{8}\left\|\nabla D^{h} \varphi\right\|^{2}+C\left\|D^{h} \varphi\right\|^{2}
$$

Combining all the previous estimates, we find the differential inequality

$$
\frac{1}{2} \frac{\mathrm{d}}{\mathrm{d} t}\left\|D^{h} \varphi\right\|^{2}+\frac{\alpha}{2}\left\|\nabla D^{h} \varphi\right\|^{2} \leqslant C\left(1+\|\nabla \varphi\|_{\mathbf{L}^{4}(\Omega)}^{4}\right)\left\|D^{h} \varphi\right\|^{2}
$$

almost everywhere in $\left(\frac{\sigma}{2}, \infty\right)$. Thanks to theorem 4.2 , an application of the uniform Gronwall lemma leads to

$$
\left\|D^{h} \varphi(t)\right\|+\left\|\nabla D^{h} \varphi\right\|_{L^{2}(t, t+1 ; \mathbf{H})} \leqslant C, \quad \forall t \geqslant \sigma .
$$

Here $C$ is a positive constant depending on $\sigma$. A final passage to the limit as $h \rightarrow 0$, together with (4.1), entails

$$
\left\|\varphi_{t}\right\|_{L^{\infty}(\sigma, \infty ; H)}+\left\|\boldsymbol{u}_{t}\right\|_{L^{\infty}\left(\sigma, \infty ; \mathbf{H}_{\sigma}\right)} \leqslant C
$$

and

$$
\left\|\varphi_{t}\right\|_{L^{2}(t, t+1 ; V)} \leqslant C, \quad \forall t \geqslant \sigma .
$$

Then, using (4.14) together with (4.13) and (5.6), we infer that, for any $p>2$, there exists $C=C(p)>0$ such that

$$
\|\mu\|_{L^{\infty}\left(\sigma, \infty ; W^{1, p}(\Omega)\right)} \leqslant C .
$$

Thanks to (4.15), we end up with

$$
\|\varphi\|_{L^{\infty}\left(\sigma, \infty ; W^{1, p}(\Omega)\right)} \leqslant C .
$$

Now, by (3.47) and (5.7), we get

$$
\|\boldsymbol{u} \cdot \nabla \varphi\|_{L^{\infty}(\sigma, \infty ; H)} \leqslant C .
$$

Thus, on account of (5.6), the elliptic regularity theory of the Neumann problem yields

$$
\|\mu\|_{L^{\infty}\left(\sigma, \infty ; H^{2}(\Omega)\right)} \leqslant C .
$$

In light of the regularity $\varphi \in \mathcal{C}(0, T ; H)$, for any $T>0$, and thanks to the validity of the separation property, it is immediate to observe by a comparison argument that $\mu \in \mathcal{C}([\sigma, T)$; $H)$, for any $T>0$. As a consequence, we have

$$
\sup _{t \geqslant \sigma}\|\mu(t)\|_{H^{2}(\Omega)} \leqslant C .
$$


Let us now assume that $J$ satisfies (H.3). Thanks to this, we have the following estimates

$$
\left\|\partial_{i}\left(\partial_{j} J * \varphi\right)\right\|_{L^{p}(\Omega)} \leqslant C(p)\|\varphi\|_{L^{p}(\Omega)}, \quad \forall p \in(1, \infty)
$$

for all $i, j \in\{1,2\}$. Since $\mu \in L^{\infty}\left(\sigma, \infty ; H^{2}(\Omega)\right)$, we easily infer from (5.8) that

$$
\left\|F^{\prime}(\varphi)\right\|_{L^{\infty}\left(\sigma, \infty ; H^{2}(\Omega)\right)} \leqslant C \text {. }
$$

Using once more the separation property and the classical result on composition of functions in Sobolev spaces, we deduce that

$$
\|\varphi\|_{L^{\infty}\left(\sigma, \infty ; H^{2}(\Omega)\right)} \leqslant C \text {. }
$$

Finally, by (3.49) and the above estimate, we reach

$$
\|\nabla \times \boldsymbol{u}\|_{L^{\infty}(\sigma, \infty ; \mathbf{V})} \leqslant C
$$

which, in turn, gives

$$
\|\boldsymbol{u}\|_{L^{\infty}\left(\sigma, \infty ; \mathbf{H}^{2}(\Omega)\right)} \leqslant C .
$$

To conclude the proof, we observe that the estimates regarding $\boldsymbol{u}$ and $\varphi$ holds for all $t \geqslant \sigma$ on account of (5.6), thus concluding the proof.

\section{Longtime behavior}

In this section we provide a description on the asymptotic behavior of solutions as time goes to $\infty$. This can be regarded as an application of our regularity results. In the first part we define the semigroup map related to problem (1.6) and (1.7) and we show the existence of the global attractor. In the second part we prove that any weak solution does converge to a single equilibrium in dimension two, taking advantage of the uniform separation property.

\subsection{The global attractor}

We define a semigroup on a suitable phase space as a consequence of theorem 3.1. Indeed, for any $\varphi_{0} \in L^{\infty}(\Omega)$ such that $\left\|\varphi_{0}\right\|_{L^{\infty}(\Omega)} \leqslant 1$ and $\left|\bar{\varphi}_{0}\right|<1$, there exists a unique global-in-time weak solution $(\boldsymbol{u}, P, \varphi)$. Then, for any given $m \in[0,1)$, we define

$$
\mathcal{H}_{m}=\left\{\varphi \in L^{\infty}(\Omega):\|\varphi\|_{L^{\infty}(\Omega)} \leqslant 1 \text { and }|\bar{\varphi}|=m\right\},
$$

and we equip it with the metric

$$
\mathbf{d}\left(\varphi_{1}, \varphi_{2}\right)=\left\|\varphi_{1}-\varphi_{2}\right\| .
$$

Then, for any $\varphi_{0} \in \mathcal{H}_{m}$, we set

$$
\varphi(t):=\mathbb{S}(t) \varphi_{0},
$$

$\varphi$ being the unique global-in-time weak solution to (1.6) and (1.7). It is immediate to check that the one-parameter family of maps $\mathbb{S}(t)$ on $\mathcal{H}_{m}$ satisfies the semigroup properties (see [44]). Moreover, we also deduce that $t \mapsto \mathbb{S}(t) \varphi_{0} \in \mathcal{C}\left([0, \infty), \mathcal{H}_{m}\right)$, for every $\varphi_{0} \in \mathcal{H}_{m}$. On account of theorem 3.2, we have a continuous dependence estimate with respect to the initial 
data in a dual norm. Nevertheless, appealing to the instantaneous regularity, we are able to show the following property

Proposition 6.1. For any $t \geqslant 0, \mathbb{S}(t) \in \mathcal{C}\left(\mathcal{H}_{m}, \mathcal{H}_{m}\right)$.

Proof. The case $t=0$ is trivial. We consider $t>0$ and a sequence $\left\{\varphi_{0 n}\right\} \subset \mathcal{H}_{m}$ such that $\mathbf{d}\left(\varphi_{0 n}, \varphi_{0}\right) \rightarrow 0$, with $\varphi_{0} \in \mathcal{H}_{m}$. Due to theorem 4.2, we infer that

$$
\|\varphi(t)\|_{V}+\left\|\varphi_{n}(t)\right\|_{V} \leqslant C \text {. }
$$

Hence, by interpolation and using (3.50), we obtain

$$
\mathbf{d}\left(\varphi_{n}(t), \varphi(t)\right) \leqslant\left\|\varphi_{n}(t)-\varphi(t)\right\|_{V^{\prime}}^{\frac{1}{2}}\left\|\varphi_{n}(t)-\varphi(t)\right\|_{V}^{\frac{1}{2}} \leqslant C\left\|\varphi_{0 n}-\varphi_{0}\right\|_{V^{\prime}}^{\frac{1}{2}} \leqslant C \mathbf{d}\left(\varphi_{0 n}, \varphi_{0}\right)^{\frac{1}{2}} .
$$

The claim follows.

The existence of the global attractor is given by

Theorem 6.1. The dynamical system $\left(\mathcal{H}_{m}, \mathbb{S}(t)\right)$ has a connected global attractor $\mathcal{A}_{m}$ bounded in $V$.

Proof. Observe that, on account of theorem 4.2, there exists a positive constant $C$, independent of the initial datum, such that

$$
\sup _{t \geqslant 1}\|\varphi(t)\|_{V} \leqslant C
$$

This entails the existence of a compact absorbing set in $\mathcal{H}_{m}$. The existence of the global attractor is thus implied by [44, theorem 1.1].

Remark 6.1. It is worth mentioning that the global attractor is more regular in the two dimensional case. Indeed, by theorem 5.2, it follows that $\mathcal{A}_{m}$ is bounded in $H^{2}(\Omega)$. Furthermore, due to theorem 5.1, the finite dimensionality of the global attractor and the existence of exponential attractors can also be proved. We refer the reader to [24] and the references therein.

\subsection{Convergence to equilibrium in dimension two}

We focus here on the longtime behavior of the single trajectory. Thanks to the separation property, we are able to employ the general strategy based on the Lyapunov property of the energy associated to the system (see proposition 3.1) and the well-known Łojasiewicz-Simon inequality. We refer the reader to [27] for the local case (see also [1, 2]).

Given an admissible initial condition $\varphi_{0} \in \mathcal{H}_{m}$, let $(\boldsymbol{u}, P, \varphi)$ be the related unique globalin-time weak solution. We introduce the $\omega$-limit set associated to $\varphi_{0}$ by

$$
\omega\left(\varphi_{0}\right)=\left\{\tilde{\varphi} \in \mathcal{H}_{m}: \exists t_{n} \rightarrow \infty \text { such that } \varphi\left(t_{n}\right) \rightarrow \tilde{\varphi} \text { in } H\right\} .
$$

On account of theorem 4.2, any weak solution is uniformly bounded in $V$. By the compact embedding results in Sobolev spaces, we deduce that the set $\omega\left(\varphi_{0}\right)$ is non-empty, compact, connected in $\mathcal{H}_{m}$. We now proceed to characterize $\omega\left(\varphi_{0}\right)$. To this aim, we introduce the notion of equilibrium point (or stationary solution) associated to (1.6) and (1.7).

Definition 6.1. A triple $\left(\boldsymbol{u}_{\infty}, P_{\infty}, \varphi_{\infty}\right)$ is called equilibrium (stationary solution) to problem( 1.6) and (1.7) if $\boldsymbol{u}_{\infty}=\mathbf{0}, P_{\infty}=\mu_{\infty} \varphi_{\infty}$ and $\varphi_{\infty} \in \mathcal{H}_{m} \cap V$ satisfies the stationary 
nonlocal Cahn-Hilliard equation

$$
F^{\prime}\left(\varphi_{\infty}\right)-J * \varphi_{\infty}=\mu_{\infty}, \quad \text { in } \Omega
$$

where $\mu_{\infty} \in \mathbb{R}$

The existence of a solution to (6.1) can be easily proven by means of a fixed point theorem. Instead, uniqueness does not hold in general (see, e.g. [4]). Besides, any stationary solution fulfils the separation property.

Lemma 6.1. For any $\varphi_{\infty} \in V \cap \mathcal{H}_{\kappa}$ satisfying (6.1), there exists $\delta>0$ such that

$$
\left\|\varphi_{\infty}\right\|_{L^{\infty}(\Omega)} \leqslant 1-\delta .
$$

Proof. Let us observe that $J * \varphi_{\infty}+\mu_{\infty} \in L^{\infty}(\Omega)$. Hence, $F^{\prime}\left(\varphi_{\infty}\right)$ is essentially bounded. Due to the assumption (H.2), the conclusion follows.

Let us introduce the set of all stationary points of the nonlocal Cahn-Hilliard equation, namely,

$$
\mathcal{S}=\left\{\varphi_{\infty} \in \mathcal{H}_{m} \cap V: \varphi_{\infty} \text { satisfies (6.1) }\right\}
$$

We now claim that $\omega\left(\varphi_{0}\right)$ consists of stationary states, namely $\omega\left(\varphi_{0}\right) \subset \mathcal{S}$. To show this, we consider a sequence $t_{n} \rightarrow \infty$ such that $\varphi\left(t_{n}\right) \rightarrow \tilde{\varphi}$, where $\tilde{\varphi} \in \omega\left(\varphi_{0}\right)$. By proposition 3.1 we observe that $\boldsymbol{u} \in L^{2}\left(0, \infty ; \mathbf{H}_{\sigma}\right)$. In light of theorem 4.2, an application of [21, Lemma 1] entails $\boldsymbol{u}(t) \rightarrow \mathbf{0}$ as $t \rightarrow \infty$. Next, for $n \in \mathbb{N}$, we define the sequence of trajectories $\left(\boldsymbol{u}_{n}(t), \varphi_{n}(t)\right)=\left(\boldsymbol{u}\left(t+t_{n}\right), \varphi\left(t+t_{n}\right)\right)$. Thanks to Theorem 4.2, $\varphi_{n} \rightarrow \varphi^{*}$ weakly star in $L^{\infty}(0, \infty ; V)$ (up to a subsequence), where $\varphi^{*}$ is a solution to (3.1) and (3.2) with $\boldsymbol{u}^{*}(t)=\mathbf{0}$, for all $t \geqslant 0$, and $\varphi^{*}(0)=\tilde{\varphi}$. Moreover, we have $\lim _{n \rightarrow \infty} \mathcal{E}\left(\varphi_{n}(t)\right)=\mathcal{E}\left(\varphi^{*}(t)\right)$, for all $t \geqslant 0$. Then, by (3.53), $\lim _{t \rightarrow \infty} \mathcal{E}(\varphi(t))=\mathcal{E}_{\infty}$ and we deduce that $\mathcal{E}\left(\varphi^{*}(t)\right)$ is constant. Thus, by (3.53) we also infer that $\nabla \mu^{*}=0$ and $\varphi_{t}^{*}=0$ for almost every $t \geqslant 0$. In turn, this implies that $\varphi^{*}(t)=\tilde{\varphi}$ for all $t \geqslant 0$. Thus, $\tilde{\varphi}$ satisfies (6.1) and the pressure is recovered through the Darcy's law.

We conclude this section by proving that $\omega\left(\varphi_{0}\right)$ is a singleton $\varphi_{\infty}$. We report here the main tool to prove the convergence to equilibrium, that is the well-known Łojasiewicz-Simon inequality (see [23])

Proposition 6.2. Let $P_{0}: H \rightarrow L_{0}^{2}$ be the projector operator. Assume that $F$ satisfies (H.2) and is real analytic in $(-1,1), \varphi \in V \cap L^{\infty}(\Omega)$ is such that $-1+\gamma \leqslant \varphi(x) \leqslant 1-\gamma$, $\forall x \in \bar{\Omega}$, for some $\gamma \in(0,1)$, and $\varphi_{\infty} \in \mathcal{S}$. Then, there exist $\theta \in\left(0, \frac{1}{2}\right), \eta>0$ and a positive constant $C$ such that

$$
\left|\mathcal{E}(\varphi)-\mathcal{E}\left(\varphi_{\infty}\right)\right|^{1-\theta} \leqslant C\left\|P_{0}\left(F^{\prime}(\varphi)-J * \varphi\right)\right\|_{*},
$$

whenever $\left\|\varphi-\varphi_{\infty}\right\| \leqslant \eta$.

The main result of this section is the following

Theorem 6.2. Assume that $F$ is real analytic on $(-1,1)$. Then, any weak solution $(\boldsymbol{u}, P, \varphi)$ converges to an equilibrium $\left(\mathbf{0}, P_{\infty}, \varphi_{\infty}\right)$. In particular, we have

$$
\lim _{t \rightarrow \infty}\|\boldsymbol{u}(t)\|+\left\|\varphi(t)-\varphi_{\infty}\right\|=0 .
$$


Proof. Thanks to the energy identity (3.53), it follows that $\mathcal{E}(\varphi(t))$ is non increasing, $\mathcal{E}(\varphi(t)) \geqslant \mathcal{E}\left(\varphi_{\infty}\right)$ and $\mathcal{E}(\varphi(t)) \rightarrow M$, where $M=\mathcal{E}\left(\varphi_{\infty}\right)$, for any $\varphi_{\infty} \in \omega\left(\varphi_{0}\right)$. Without loss of generality, we consider $\mathcal{E}(\varphi(t))>\mathcal{E}\left(\varphi_{\infty}\right)$, for all $t \geqslant 0$. Otherwise, if there exists $\bar{t}>0$ such that $\mathcal{E}(\varphi(t))=\mathcal{E}\left(\varphi_{\infty}\right)$, it is evident that $\varphi(t)=\varphi(\bar{t})$, for all $t \geqslant \bar{t}$, and the claim follows. On the other hand, we fix $\theta \in\left(0, \frac{1}{2}\right)$ and $\eta>0$ given by proposition 6.2. Via a contradiction argument, it is possible to show that there exists $t^{*}>0$ such that $\left\|\varphi(t)-\varphi_{\infty}\right\| \leqslant \eta$, for all $t \geqslant t^{*}$ (see, e.g. [21]). Then, by theorem 5.1 and proposition 6.2 , for any $t \geqslant t^{*}$, we have

$$
\begin{aligned}
\left(\mathcal{E}(\varphi)-\mathcal{E}\left(\varphi_{\infty}\right)\right)^{1-\theta} & \leqslant\left(C\left\|P_{0}\left(F^{\prime}(\varphi)-J * \varphi\right)\right\|_{*}^{\frac{1}{1-\theta}}\right)^{1-\theta} \\
& \leqslant C\|\nabla \mu\| .
\end{aligned}
$$

By using (3.53) together with the above inequalities, we deduce that

$$
\begin{aligned}
-\frac{\mathrm{d}}{\mathrm{d} t}\left(\mathcal{E}(\varphi)-\mathcal{E}\left(\varphi_{\infty}\right)\right)^{\theta} & =-\theta\left(\mathcal{E}(\varphi)-\mathcal{E}\left(\varphi_{\infty}\right)\right)^{\theta-1} \frac{\mathrm{d}}{\mathrm{d} t} \mathcal{E}(\varphi) \\
& \geqslant \frac{\theta\left(\|\boldsymbol{u}\|^{2}+\|\nabla \mu\|^{2}\right)}{C\|\nabla \mu\|} \geqslant C\|\nabla \mu\| .
\end{aligned}
$$

An integration on the time interval $\left(t^{*}, \infty\right)$, for $t^{*}$ sufficiently large, leads to $\nabla \mu \in L^{1}\left(t^{*}, \infty ; \mathbf{H}\right)$. Also, in light of (3.4) and (3.55), we obtain $\boldsymbol{u} \in L^{1}\left(t^{*}, \infty ; \mathbf{H}_{\sigma}\right)$. By comparison, we find $\varphi_{t} \in L^{1}\left(t^{*}, \infty ; V^{\prime}\right)$. Thus, we conclude that $\varphi(t)$ converges in $V^{\prime}$ as $t$ goes to $\infty$. Using the interpolation $\|v\| \leqslant C\|v\|_{V^{\prime}}^{\frac{1}{2}}\|v\|_{V}^{\frac{1}{2}}$ and the uniform bound in $V$ (see theorem 4.2), we deduce (6.3).

\section{Conclusions and further developments}

The main purpose of this paper is to address the well-posedness of the nonlocal CahnHilliard-Hele-Shaw system with logarithmic potential in dimension three and the global regularity problem of its solutions. As mentioned in the introduction, we point out that this analysis has been carried out for the corresponding local Cahn-Hilliard-Hele-Shaw system in dimension two only (see [27]). In our opinion, there are several interesting open issues to be investigated. First of all, in connection to the application of diffuse interface models to tumor growth dynamics, the results herein could be extended to the case with a non-autonomous mass source term accounting for cell proliferation (see, for instance, [36, 43, 48]). In particular, the second and third equations in (1.6) read now as follows

$$
\operatorname{div} \boldsymbol{u}=S, \quad \varphi_{t}+u \cdot \nabla \varphi-\Delta \mu=S,
$$

where $S$ can be a given source or it can depend on other state variables (e.g. nutrients). A further problem is concerned with the inclusion of a body force $\mathbf{G}$ (that may depend on the density, see [16, 38]) or a Coriolis force (see [10]) in the Darcy's law. A further important issue would be the so-called unmatched viscosities case, i.e. to allow the viscosity to depend

on the concentration. In this case, the Darcy's law can be written as $\nu(\varphi) \boldsymbol{u}=-\nabla P+\mu \nabla \varphi$, where $\nu:[0,1] \rightarrow \mathbb{R}$ is a strictly positive and bounded function. We recall that the unmatched viscosity case has been recently analyzed in [14] for the Cahn-Hilliard-Brinkman system. The nonlocal version of such a system also presents several open issues in terms of uniqueness and regularity. We already pointed out that the Cahn-Hilliard-Hele-Shaw (or CahnHilliard-Darcy) system also plays a role in modeling porous media. In this context, it would 
be interesting to consider nonlocal versions of models for karstic geometry (see [35]). Finally, we recall that very few recent papers are devoted to the numerical analysis of nonlocal CahnHilliard type equations (see [29-31]). We think that it would be worth extending this analysis, for instance, to the present model and then compare the numerical simulations with the ones existing for the local model (e.g. [11, 18, 32, 34, 40, 49]) possibly with the logarithmic potential (e.g. [12] and the references therein). Numerics could show, for instance, whether there are detectable differences between local and nonlocal models or it could give some hints about the possible validity of the separation property in three dimensions. This property seems related to the diffusion effects and numerical simulations might also shed some light on this relation.

\section{Acknowledgments}

The work of the first author was supported by the Engineering and Physical Sciences Research Council [EP/L015811/1]. The second and third author are members of the Gruppo Nazionale per l'Analisi Matematica, la Probabilità e le loro Applicazioni (GNAMPA) of the Istituto Nazionale di Alta Matematica (INdAM). Their work was supported by GNAMPA-INdAM Project 'Analisi matematica di modelli a interfaccia diffusa per fluidi complessi'.

\section{ORCID iDs}

Francesco Della Porta (1) https://orcid.org/0000-0002-3090-5770

Andrea Giorgini (․ https://orcid.org/0000-0002-5908-1306

Maurizio Grasselli (1) https://orcid.org/0000-0003-2521-2926

\section{References}

[1] Abels H 2009 On a diffuse interface model for two-phase flows of viscous, incompressible fluids with matched densities Arch. Ration. Mech. Anal. 194 463-506

[2] Abels H and Wilke M 2007 Convergence to equilibrium for the Cahn-Hilliard equation with a logarithmic free energy Nonlinear Anal. 67 3176-93

[3] Anderson D M, McFadden G B and Wheeler A A 1998 Diffuse-interface methods in fluid mechanics Annu. Rev. Fluid Mech. 30 139-65

[4] Bates P W and Chmaj A 1999 An integrodifferential model for phase transitions: stationary solutions in higher space dimensions J. Stat. Phys. 95 1119-39

[5] Bedrossian J, Rodríguez N and Bertozzi A 2011 Local and global well-posedness for an aggregation equation and Patlak-Keller-Segel models with degenerate diffusion Nonlinearity 24 1683-714

[6] Bosia S, Conti M and Grasselli M 2015 On the Cahn-Hilliard-Brinkman system Commun. Math. Sci. 13 1541-67

[7] Brezis H 1973 Opérateurs Maximaux Monotones et Semi-groupes de Contractions dans les Espaces de Hilbert (North-Holland Mathematics Studies vol 5) (Amsterdam: North-Holland)

[8] Cahn J W and Hilliard J E 1958 Free energy of a nonuniform system. I. Interfacial free energy J. Chem. Phys. 28 258-67

[9] Cherfils L, Miranville A and Zelik S 2011 The Cahn-Hilliard equation with logarithmic potentials Milan J. Math. 79 561-96

[10] Chen C-Y, Huang Y-S and Miranda J A 2011 Diffuse-interface approach to rotating Hele-Shaw flows Phys. Rev. E 84046302

[11] Chen W, Liu Y, Wang C and Wise S M 2016 Convergence analysis of a fully discrete finite difference scheme for the Cahn-Hilliard-Hele-Shaw equation Math. Comput. 85 2231-57

[12] Chen W, Wang C, Wang X and Wise S M 2017 A positivity-preserving, energy stable numerical scheme for the Cahn-Hilliard equation with logarithmic potential (arXiv:1712.03225v1 [math.

NA]) 
[13] Colli P, Krejčí P, Rocca E and Sprekels J 2007 Nonlinear evolution inclusions arising from phase change models Czech. Math. J. 57 1067-98

[14] Conti M and Giorgini A 2018 The three-dimensional Cahn-Hilliard-Brinkman system with unmatched viscosities (hal-01559179v2)

[15] Dai M, Feireisl E, Rocca E, Schimperna G and Schonbek M E 2017 Analysis of a diffuse interface model of multispecies tumor growth Nonlinearity 30 1639-58

[16] Dedé L, Garcke H and Lam K F 2018 A Hele-Shaw-Cahn-Hilliard model for incompressible twophase flows with different densities J. Math. Fluid Mech. 20 531-67

[17] Della Porta F and Grasselli M 2016 On the nonlocal Cahn-Hilliard-Brinkman and Cahn-HilliardHele-Shaw systems Commun. Pure Appl. Anal. 15 299-317

Della Porta F and Grasselli M 2017 On the nonlocal Cahn-Hilliard-Brinkman and Cahn-HilliardHele-Shaw systems Commun. Pure Appl. Anal. 16 369-72 (erratum)

[18] Feng X-B and Wise S 2012 Analysis of a Darcy-Cahn-Hilliard diffuse interface model for the Hele-Shaw flow and its fully discrete finite element approximation SIAM J. Numer. Anal. 50 1320-43

[19] Frigeri S, Gal C G and Grasselli M 2016 On nonlocal Cahn-Hilliard-Navier-Stokes systems in two dimensions J. Nonlinear Sci. 26 847-93

[20] Frigeri S and Grasselli M 2012 Nonlocal Cahn-Hilliard-Navier-Stokes systems with singular potential Dyn. PDE 9 273-304

[21] Frigeri S, Grasselli M and Krex̌í P 2013 Strong solutions for two-dimensional nonlocal Cahn Hilliard-Navier-Stokes systems J. Differ. Equ. 255 2587-614

[22] Frigeri S, Grasselli M and Rocca E 2015 A diffusive interface model for two-phase incompressible flows with nonlocal interactions and nonconstant mobility Nonlinearity 28 1257-93

[23] Gajewski H and Griepentrog J A 2006 A descent method for the free energy of multicomponent systems Discrete Contin. Dyn. Syst. 15 505-28

[24] Gal C G, Giorgini A and Grasselli M 2017 The nonlocal Cahn-Hilliard equation with singular potential: well-posedness, regularity and strict separation property J. Differ. Equ. 263 5253-97

[25] Giacomin G and Lebowitz J L 1997 Phase segregation dynamics in particle systems with long range interactions. I. Macroscopic limits J. Stat. Phys. 87 37-61

[26] Giga Y and Miyakawa T 1985 Solutions in $L^{r}$ of the Navier-Stokes initial value problem Arch. Ration. Mech. Anal. 89 267-81

[27] Giorgini A, Grasselli M and Wu H 2018 The Cahn-Hilliard-Hele-Shaw system with singular potential Ann. Inst. Henri Poincaré Anal. Non Linéaire 35 1079-118

[28] Girault V and Raviart P-A 1986 Finite Element Methods for Navier-Stokes Equations. Theory and Algorithms (Springer Series in Computational Mathematics vol 5) (Berlin: Springer)

[29] Guan Z, Lowengrub J S, Wang C and Wise S M 2014 Second order convex splitting schemes for periodic nonlocal Cahn-Hilliard and Allen-Cahn equations J. Comput. Phys. 277 48-71

[30] Guan Z, Wang C and Wise S M 2014 A convergent convex splitting scheme for the periodic nonlocal Cahn-Hilliard equation Numer. Math. 128 377-406

[31] Guan Z, Lowengrub J S and Wang C 2017 Convergence analysis for second-order accurate schemes for the periodic nonlocal Allen-Cahn and Cahn-Hilliard equations Math. Methods Appl. Sci. 40 6836-63

[32] Guo R, Xia Y and Xu Y 2014 An efficient fully-discrete local discontinuous Galerkin method for the Cahn-Hilliard-Hele-Shaw system J. Comput. Phys. 264 23-40

[33] Han D, Sun D and Wang X 2014 Two-phase flows in karstic geometry Math. Methods Appl. Sci. 37 3048-63

[34] Han D 2016 A decoupled unconditionally stable numerical scheme for the Cahn-Hilliard-HeleShaw system J. Sci. Comput. 66 1102-21

[35] Han D, Wang X and Wu H 2014 Existence and uniqueness of global weak solutions to a CahnHilliard-Stokes-Darcy system for two phase incompressible flows in karstic geometry J. Differ. Equ. 257 3887-933

[36] Jiang J, Wu H and Zheng S 2015 Well-posedness and long-time behavior of a non-autonomous Cahn-Hilliard-Darcy system with mass source modeling tumor growth J. Differ. Equ. $2593032-77$

[37] Lee D, Huh J-Y, Jeong D, Shin J, Yun A and Kim J 2014 Physical, mathematical, and numerical derivations of the Cahn-Hilliard equation Comput. Math. Sci. 81 216-25

[38] Lee H-G, Lowengrub J S and Goodman J 2002 Modeling pinchoff and reconnection in a Hele-Shaw cell. I. The models and their calibration Phys. Fluids 14 492-513 
[39] Lions J-L 1961-1962 Quelques Remarques sur les Problèmes de Dirichlet et de Neumann (Séminaire Jean Leray) (Paris: Collége de France) pp 1-18

[40] Liu Y, Chen W, Wang C and Wise S M 2017 Error analysis of a mixed finite element method for a Cahn-Hilliard-Hele-Shaw system Numer. Math. 135 679-709

[41] Lowengrub J, Goodman J, Lee H, Longmire E, Shelley M and Truskinovsky L 1999 Topological Transitions in Liquid/Liquid Interfaces (Chapman \& Hall/CRC Research Notes in Mathematics, London vol 409) pp 221-36

[42] Lowengrub J and Truskinovsky L 1998 Quasi-incompressible Cahn-Hilliard fluids and topological transitions Proc. R. Soc. A 454 2617-54

[43] Lowengrub J, Titi E and Zhao K 2013 Analysis of a mixture model of tumor growth Eur. J. Appl. Math. 24 1-44

[44] Temam R 1997 Infinite-Dimensional Dynamical Systems in Mechanics and Physics 2nd edn (Berlin: Springer)

[45] Vershueren M, van de Vosse F N and Meijer H E H 2001 Diffuse-interface modelling of thermocapillary flow instabilities in a Hele-Shaw cell J. Fluid Mech. 434 153-66

[46] Wang X-M and Wu H 2012 Long-time behavior for the Hele-Shaw-Cahn-Hilliard system Asymptotic Anal. 78 217-45

[47] Wang X-M and Zhang Z-F 2013 Well-posedness of the Hele-Shaw-Cahn-Hilliard system Ann. Inst. Henri Poincaré Anal. Non Linéaire 30 367-84

[48] Wise S M, Lowengrub J S, Frieboes H B and Cristini V 2008 Three-dimensional multispecies nonlinear tumor growth-I: model and numerical method J. Theor. Biol. 253 524-43

[49] Wise S M 2010 Unconditionally stable finite difference, nonlinear multigrid simulation of the Cahn-Hilliard-Hele-Shaw system of equations J. Sci. Comput. 44 38-68 